\title{
THE NEXT GENERATION ATLAS OF QUASAR SPECTRAL ENERGY DISTRIBUTIONS FROM RADIO TO X-RAYS
}

\author{
Zhaohui Shang ${ }^{1,2}$, Michael S. Brotherton ${ }^{2}$, Beverley J. Wills ${ }^{3}$, D. Wills ${ }^{3}$, Sabrina L. Cales ${ }^{2}$, Daniel A. Dale ${ }^{2}$, \\ Richard F. Green ${ }^{4}$, Jessie C. Runnoe ${ }^{2}$, Rodrigo S. Nemmen ${ }^{5}$, Sarah C. Gallagher ${ }^{6}$, Rajib Ganguly ${ }^{7}$, Dean C. Hines ${ }^{8}$, \\ Benjamin J. Kelly ${ }^{2}$, Gerard A. Kriss ${ }^{9}, 10$, Jun LI$^{1}$, Baitian Tang ${ }^{11,12}$, and YanXia Xie ${ }^{1,13}$ \\ ${ }^{1}$ Department of Physics, Tianjin Normal University, Tianjin 300387, China; zshang @ gmail.com \\ ${ }^{2}$ Department of Physics and Astronomy, University of Wyoming, Laramie, WY 82071, USA \\ ${ }^{3}$ Department of Astronomy, University of Texas at Austin, 1 University Station, C1400 Austin, TX 78712, USA \\ ${ }^{4}$ Large Binocular Telescope Observatory, University of Arizona, 933 North Cherry Avenue, Tucson, AZ 85721, USA \\ ${ }^{5}$ NASA/Goddard Space Flight Center, Greenbelt, MD 20771, USA \\ ${ }^{6}$ Department of Physics and Astronomy, The University of Western Ontario, London, ON N6A 3K7, Canada \\ ${ }^{7}$ Department of Computer Science, Engineering, \& Physics, University of Michigan-Flint, 213 Murchie Science Building, \\ 303 Kearsley Street, Flint, MI 48502, USA \\ ${ }^{8}$ Space Science Institute, 4750 Walnut Street, Suite 205, Boulder, CO 80301, USA \\ ${ }^{9}$ Space Telescope Science Institute, 3700 San Martin Drive, Baltimore, MD 21218, USA \\ ${ }^{10}$ Department of Physics and Astronomy, The Johns Hopkins University, Baltimore, MD 21218, USA \\ ${ }^{11}$ Department of Physics, 1245 Webster Hall, Washington State University, Pullman, WA 99164-2814, USA \\ ${ }^{12}$ Department of Astronomy, Nanjing University, Nanjing 210093, China \\ ${ }^{13}$ Shanghai Astronomical Observatory, Chinese Academy of Science, 80 Nandan Road, Shanghai 200030, China \\ Received 2011 April 21; accepted 2011 July 8; published 2011 August 17
}

\begin{abstract}
We have produced the next generation of quasar spectral energy distributions (SEDs), essentially updating the work of Elvis et al. by using high-quality data obtained with several space- and ground-based telescopes, including NASA's Great Observatories. We present an atlas of SEDs of 85 optically bright, non-blazar quasars over the electromagnetic spectrum from radio to X-rays. The heterogeneous sample includes 27 radio-quiet and 58 radio-loud quasars. Most objects have quasi-simultaneous ultraviolet-optical spectroscopic data, supplemented with some far-ultraviolet spectra, and more than half also have Spitzer mid-infrared Infrared Spectrograph spectra. The X-ray spectral parameters are collected from the literature where available. The radio, far-infrared, and near-infrared photometric data are also obtained from either the literature or new observations. We construct composite SEDs for radio-loud and radio-quiet objects and compare these to those of Elvis et al., finding that ours have similar overall shapes, but our improved spectral resolution reveals more detailed features, especially in the mid- and near-infrared.
\end{abstract}

Key words: atlases - galaxies: active - infrared: galaxies - quasars: general - radio continuum: galaxies ultraviolet: galaxies - X-rays: galaxies

Online-only material: color figures, extended figure, machine-readable table, supplementary data

\section{INTRODUCTION}

The supermassive black holes powering quasars (or active galactic nuclei, AGNs) do not themselves shine. It is the heated material surrounding the black holes that emits the radiation signatures of quasars. These signatures include broad emission lines characteristic of high-velocity gas moving at thousands of kilometers per second, and extremely high continuum luminosity in excess of that of entire galaxies. Continuum emission is seen in all parts of the electromagnetic spectrum from the highest energies (gamma rays and X-rays) to the lowest (radio waves). The power emitted is similarly high, within an order of magnitude or so, over much of this range, although there can be significant variation from quasar to quasar.

There are no single states of matter or single processes capable of reproducing the spectral energy distribution (SED) of a quasar. A combination of both thermal and non-thermal processes has been invoked to explain, at different parts of the SED, the emission from gas in a variety of states, at a variety of temperatures, at a variety of distances, and experiencing a variety of environments. Quasars seem to have many different components that are expressed in different parts of the electromagnetic spectrum.

So what are the components of the quasar? The central supermassive black hole is the ultimate engine, allowing the liberation of gravitational potential energy. The primary source of electromagnetic emission is likely an accretion disk formed of hot gas spiraling into the black hole and shining in the optical through ultraviolet (UV). A hot atmosphere upscatters the disk photons to X-ray energies. A jet shooting out from the inner accretion disk emits synchrotron radiation, dominating radio emission and sometimes higher energies. An obscuring torus of relatively cool gas and dust, heated by photons from the accretion disk, thermally radiates in the near-infrared (NIR) and mid-infrared (MIR). The far-infrared (FIR) part of the SED comes from cooler dust, perhaps distributed throughout the host galaxy, that may be heated by stars rather than the quasar itself. Other regions in and among these continuum-emitting parts are responsible for the prominent emission lines present in quasar spectra. The details of all these parts, mechanisms, and their relationships are not yet completely understood because multiwavelength data to further our understanding have been difficult to gather.

Multiwavelength astronomy is challenging. No single telescope can observe all wavelengths. Many parts of the electromagnetic spectrum cannot penetrate the Earth's atmosphere and require space-based observations. Quasars are variable and this means that simultaneous or nearly simultaneous observations are desirable, at least in some parts of the spectrum. Different technologies have different sensitivity levels and what may be 
an easily observed target at one wavelength may be difficult to detect at another.

There were a number of pioneering works on quasar SEDs in the 1980s (e.g., Edelson \& Malkan 1986; Ward et al. 1987; Kriss 1988; Sanders et al. 1989; Sun \& Malkan 1989). In the 1990s, Elvis et al. (1994, hereafter E94) established the first large, high-quality atlas of quasar SEDs. The timing of their work was predicated on the launch of several space-based telescopes, notably IRAS in the mid- to far-IR, IUE in the UV, and Einstein in the X-rays, that for the first time provided observations of large numbers of quasars in these wavebands. They established the differences between the SEDs of radio-loud (RL) and radioquiet (RQ) quasars. They also characterized the variance of quasar SEDs, which is rather substantial, and explored the problem of bolometric corrections.

Since the work of E94, there have been several significant investigations of quasar SEDs (e.g., Kuraszkiewicz et al. 2003; Risaliti \& Elvis 2004). Richards et al. (2006) is the largest that covers the entire electromagnetic spectrum, including data from the Sloan Digital Sky Survey and Spitzer, supplemented by near-IR, GALEX UV, VLA radio, and ROSAT X-ray data, where available. One of their key findings was again the wide range of SED shapes, and how assuming a mean SED can potentially lead to errors in bolometric luminosities as high as $50 \%$.

Other investigations of SEDs have focused on subclasses, like broad absorption line (BAL) quasars (e.g., Gallagher et al. 2007), Two Micron All Sky Survey (2MASS) red quasars (Kuraszkiewicz et al. 2009), hard X-ray selected quasars (Polletta et al. 2000, 2007), or on individual objects (e.g., Zheng et al. 2001). More detailed SED work has been done on limited portions of the entire electromagnetic spectrum, such as the optical-X-ray (e.g., Laor et al. 1997), the FIR to optical (e.g., Netzer et al. 2007), or optical/UV to X-ray (Grupe et al. 2010).

There has also been work looking for relationships among more detailed spectral features, like emission lines and SEDs. Wilkes et al. (1999), examining 41 quasars with SED information, for instance, found a variety of Baldwin effects (Baldwin 1977), anticorrelations between emission-line equivalent width and UV luminosity, as well as some correlations between properties of Fe II and C IV emission lines and the ratio of the optical to X-ray luminosity. Schweitzer et al. (2006) and Netzer et al. (2007) studied Palomar-Green quasars with FIR to optical data and confirmed that most FIR radiation is due to star-forming activity. However, Netzer et al. (2007) also argue, based on a correlation between $L(5100 \AA)$ and $L(60 \mu \mathrm{m})$, an alternative view that a large fraction of FIR radiation could result from direct AGN heating.

Ideally what one would like in studying quasars is a complete inventory over all time and all directions of all photons emitted. The best we can do now is to obtain spectrophotometric snapshots, close in time, of some spectral regions, supplemented by photometry in other accessible wavebands, from our particular line of sight toward a quasar. The technology has improved since the 1990s, with spacecraft such as the Hubble Space Telescope (HST), Chandra and XMM, and Spitzer replacing IUE, Einstein and ROSAT, and IRAS, respectively.

This atlas is meant to update the last decade's work with a modern set of quasar SEDs using the next generation of telescopes and instruments. Whenever possible we have striven to use high-quality spectrophotometry in addition to photometry, which will enable investigations like those of Wilkes et al. (1999). One hope is that spectral features will be found that correlate with SEDs and allow their shapes to be determined without the need for complete multiwavelength observations. Any correlations found should provide deeper insight into quasars. We plan additional papers based on this data set performing various types of investigations, as well as addressing both observational and theoretical aspects of bolometric corrections.

In the next section (Section 2), we describe our sample, which is composed of three subsamples that have excellent quasisimultaneous optical through UV or far-UV (FUV) spectrophotometry serving as a starting point for SED construction. We then describe the sample properties. Subsequent sections describe the data (Section 3), starting with about $100 \mathrm{MHz}$ radio and moving to higher energies to about $10 \mathrm{keV}$ X-ray. We then discuss corrections to the SEDs, such as Galactic dereddening and host galaxy removal (Section 4). In Section 5, we present the SEDs for our quasars individually, then as composites for RL and RQ subsamples, which are known to differ significantly. We then discuss the properties of the SEDs, and compare our composites with those of E94. We finish the paper with a summary of these results, future plans, and some concluding remarks (Section 6).

\section{SAMPLE}

In the past, in order to study the optical and UV properties of quasars, we embarked on several programs of obtaining quasisimultaneous spectrophotometry utilizing various ground- and space-based telescopes. As this is a challenging endeavor, and the data we obtained were of high quality, we began the construction of SEDs with these samples, adding data at longer and shorter wavelengths. For convenience, we refer to our three subsamples by the abbreviations PGX, FUSE-HST, and RLQ. These subsamples are described below, and Table 1 provides our combined SED sample of 85 objects and their basic properties.

Several objects in the FUSE-HST subsample are also in the other two subsamples and there may be repeated observations in UV and/or optical. We choose to analyze the data with the FUSE-HST subsample because of their higher quality.

\section{1. $P G X$}

The "PGX" sample consists of 22 of 23 Palomar-Green quasars in the complete sample selected by Laor et al. (1994, 1997) from the Bright Quasar Survey (BQS; Schmidt \& Green 1983). Interested in observing the soft-X-ray regime using bright quasars, Laor et al. (1994, 1997) started with the UVexcess selected BQS and added the restrictions that $z \leqslant 0.4$ and Galactic H I column density $N_{\mathrm{H}_{\mathrm{I}}}<1.9 \times 10^{20} \mathrm{~cm}^{-2}$. We obtained low-resolution UV spectra with HST and conducted quasi-simultaneous ground-based observations at McDonald Observatory, usually within a month. Shang et al. (2007) provide additional details and constructed the UV-optical SEDs for this sample.

\subsection{FUSE-HST}

The "FUSE-HST" sample originates with the Far Ultraviolet Spectroscopic Explorer (FUSE) AGN program (Kriss 2000), which surveyed more than 100 of the UV-brightest AGNs. About 20 of these were also observed in an HST spectral snapshot survey with sufficient signal-to-noise ratio during 1999-2000. The FUSE observations were scheduled as close in time as possible with the HST snapshot observations, and ground-based optical spectra were also obtained during the same period at Kitt Peak National Observatory (KPNO). We exclude a few objects because of the lack of an optical spectrum (NGC 3783, 
Table 1

SED Sample

\begin{tabular}{|c|c|c|c|c|c|c|c|c|c|}
\hline ID & Name & Other Name & R.A. (J2000) & Decl. (J2000) & $z^{\mathrm{a}}$ & $E(B-V)^{\mathrm{b}}$ & $R^{\mathrm{c}}$ & $\lambda L_{\lambda}(3000 \AA)^{\mathrm{d}}$ & SampleID \\
\hline 1 & MC2 0042+101 & & $00: 44: 58.72$ & $+10: 26: 53.7$ & 0.5870 & 0.068 & 840 & 46.22 & RLQ \\
\hline 2 & PG $0052+251$ & & $00: 54: 52.10$ & $+25: 25: 38.0$ & 0.1544 & 0.047 & 0.34 & 46.30 & FUSE \\
\hline 3 & PKS 0112-017 & UM 310 & $01: 15: 17.10$ & $-01: 27: 04.6$ & 1.3743 & 0.062 & 2819 & 45.92 & RLQ \\
\hline 5 & $3 \mathrm{C} 47$ & & $01: 36: 24.40$ & $+20: 57: 27.0$ & 0.4250 & 0.061 & 6570 & 44.94 & RLQ \\
\hline 6 & 4C 01.04 & PHL 1093 & $01: 39: 57.25$ & $+01: 31: 46.2$ & 0.2634 & 0.029 & 2556 & 45.93 & RLQ \\
\hline 7 & 4C 10.06 & PKS 0214+10 & 02:17:07.66 & $+11: 04: 10.1$ & 0.4075 & 0.109 & 472 & 45.54 & RLQ \\
\hline 10 & $3 \mathrm{C} 175$ & PKS 0710+11 & 07:13:02.40 & $+11: 46: 14.7$ & 0.7693 & 0.147 & 978 & 45.52 & RLQ \\
\hline 11 & 3C 186 & & $07: 44: 17.45$ & $+37: 53: 17.1$ & 1.0630 & 0.050 & 2131 & 45.86 & RLQ \\
\hline 12 & B2 $0742+31$ & & $07: 45: 41.67$ & $+31: 42: 56.6$ & 0.4616 & 0.068 & 591 & 45.86 & RLQ \\
\hline 13 & IRAS F07546+3928 & FBQS J075800.0+392029 & 07:58:00.05 & $+39: 20: 29.1$ & 0.0953 & 0.066 & 0.25 & 45.23 & FUSE \\
\hline 14 & $3 \mathrm{C} 207$ & & $08: 40: 47.59$ & $+13: 12: 23.6$ & 0.6797 & 0.093 & 4238 & 46.32 & RLQ \\
\hline 15 & PG 0844+349 & TON 951 & $08: 47: 42.40$ & $+34: 45: 04.0$ & 0.0643 & 0.037 & 0.07 & 45.06 & FUSE \\
\hline 21 & PG $0953+414$ & & 09:56:52.40 & $+41: 15: 22.0$ & 0.2338 & 0.013 & 0.61 & 46.16 & FUSE,PGX \\
\hline 22 & 4C 55.17 & & $09: 57: 38.18$ & $+55: 22: 57.8$ & 0.8990 & 0.009 & 5525 & 46.11 & RLQ \\
\hline 23 & $3 \mathrm{C} 232$ & & 09:58:20.95 & $+32: 24: 02.2$ & 0.5297 & 0.015 & 736 & 45.65 & RLQ \\
\hline 24 & PG $1001+054$ & & 10:04:20.09 & $+05: 13: 00.5$ & 0.1603 & 0.016 & 1.12 & 45.43 & PGX \\
\hline 25 & $4 C 22.26$ & PKS $1002+22$ & $10: 04: 45.74$ & $+22: 25: 19.4$ & 0.9760 & 0.039 & 1817 & 45.99 & RLQ \\
\hline 26 & $4 \mathrm{C} 41.21$ & & $10: 10: 27.52$ & $+41: 32: 38.9$ & 0.6124 & 0.015 & 820 & 45.31 & RLQ \\
\hline 27 & 4C 20.24 & PKS $1055+20$ & 10:58:17.90 & $+19: 51: 50.9$ & 1.1135 & 0.025 & 4152 & 46.12 & RLQ \\
\hline 28 & PG 1100+772 & 3C 249.1 & 11:04:13.69 & $+76: 58: 58.0$ & 0.3114 & 0.034 & 444 & 45.83 & FUSE,RLQ \\
\hline 29 & PG 1103-006 & PKS 1103-006 & 11:06:31.77 & $-00: 52: 52.5$ & 0.4234 & 0.044 & 868 & 45.95 & RLQ \\
\hline 30 & $3 \mathrm{C} 254$ & & $11: 14: 38.48$ & $+40: 37: 20.3$ & 0.7363 & 0.015 & 5139 & 45.08 & RLQ \\
\hline 31 & PG $1114+445$ & & 11:17:06.40 & $+44: 13: 33.0$ & 0.1440 & 0.016 & 0.11 & 45.76 & PGX \\
\hline 32 & PG 1115+407 & & $11: 18: 30.20$ & $+40: 25: 53.0$ & 0.1541 & 0.016 & 0.33 & 46.53 & PGX \\
\hline 41 & $4 C 64.15$ & & $12: 17: 41.85$ & $+64: 07: 07.8$ & 1.3000 & 0.019 & 2365 & 45.67 & RLQ \\
\hline 42 & PG 1216+069 & & 12:19:20.88 & $+06: 38: 38.4$ & 0.3319 & 0.022 & 4.64 & 43.68 & PGX \\
\hline 43 & PG 1226+023 & $3 C 273$ & 12:29:06.70 & $+02: 03: 08.6$ & 0.1576 & 0.021 & 1667 & 44.46 & FUSE,PGX,RI \\
\hline 44 & 4C 30.25 & B2 $1248+30$ & $12: 50: 25.55$ & $+30: 16: 39.3$ & 1.0610 & 0.016 & 831 & 45.98 & RLQ \\
\hline 45 & 3C 277.1 & & $12: 52: 26.35$ & $+56: 34: 19.7$ & 0.3199 & 0.010 & 3354 & 45.13 & RLQ \\
\hline 46 & PG $1259+593$ & & 13:01:12.90 & $+59: 02: 06.4$ & 0.4769 & 0.008 & 0.02 & 44.62 & FUSE \\
\hline 47 & $3 \mathrm{C} 281$ & & 13:07:54.00 & $+06: 42: 14.3$ & 0.6017 & 0.039 & 1683 & 45.06 & RLQ \\
\hline 48 & PG $1309+355$ & TON 1565 & $13: 12: 17.77$ & $+35: 15: 21.2$ & 0.1823 & 0.012 & 23.81 & 45.70 & PGX \\
\hline 49 & PG $1322+659$ & & $13: 23: 49.54$ & $+65: 41: 48.0$ & 0.1684 & 0.019 & 0.16 & 44.61 & FUSE,PGX \\
\hline 50 & $3 \mathrm{C} 288.1$ & & $13: 42: 13.18$ & $+60: 21: 42.9$ & 0.9631 & 0.018 & 2660 & 45.67 & RLQ \\
\hline 51 & PG $1351+640$ & IRAS F13517+6400 & $13: 53: 15.81$ & $+63: 45: 45.4$ & 0.0882 & 0.020 & 1.24 & 45.57 & FUSE \\
\hline 52 & B2 1351+31 & & $13: 54: 05.35$ & $+31: 39: 01.9$ & 1.3260 & 0.017 & 888 & 44.94 & RLQ \\
\hline 53 & PG $1352+183$ & & $13: 54: 35.60$ & $+18: 05: 17.2$ & 0.1510 & 0.019 & 0.24 & 44.89 & PGX \\
\hline 54 & 4C 19.44 & & $13: 57: 04.43$ & $+19: 19: 07.4$ & 0.7192 & 0.060 & 2632 & 45.42 & RLQ \\
\hline 55 & 4C 58.29 & & $13: 58: 17.63$ & $+57: 52: 04.9$ & 1.3740 & 0.010 & 453 & 44.70 & RLQ \\
\hline 56 & PG $1402+261$ & TON 182 & 14:05:16.19 & $+25: 55: 34.9$ & 0.1650 & 0.016 & 0.30 & 45.50 & PGX \\
\hline 57 & PG $1411+442$ & & $14: 13: 48.30$ & $+44: 00: 14.0$ & 0.0895 & 0.008 & 0.14 & 46.26 & PGX \\
\hline 58 & PG $1415+451$ & & 14:17:00.80 & $+44: 56: 06.0$ & 0.1143 & 0.009 & 0.27 & 46.07 & PGX \\
\hline 59 & PG $1425+267$ & TON 202 & $14: 27: 35.54$ & $+26: 32: 13.6$ & 0.3637 & 0.019 & 206 & 45.19 & PGX \\
\hline 60 & PG $1427+480$ & & $14: 29: 43.00$ & $+47: 47: 26.0$ & 0.2203 & 0.017 & 0.03 & 44.92 & PGX \\
\hline 61 & PG $1440+356$ & MRK 478 & $14: 42: 07.46$ & $+35: 26: 22.9$ & 0.0773 & 0.014 & 0.18 & 44.79 & PGX \\
\hline 62 & PG $1444+407$ & & $14: 46: 45.90$ & $+40: 35: 05.0$ & 0.2673 & 0.014 & 0.10 & 44.80 & PGX \\
\hline 63 & PG $1512+370$ & 4C 37.43 & $15: 14: 43.04$ & $+36: 50: 50.4$ & 0.3700 & 0.022 & 717 & 45.20 & PGX \\
\hline 64 & PG $1534+580$ & MRK 290 & $15: 35: 52.36$ & $+57: 54: 09.2$ & 0.0303 & 0.015 & 1.37 & 44.89 & FUSE \\
\hline 65 & PG $1543+489$ & IRAS F15439+4855 & $15: 45: 30.20$ & $+48: 46: 09.0$ & 0.4000 & 0.018 & 1.36 & 44.59 & PGX \\
\hline
\end{tabular}


Table 1

(Continued)

\begin{tabular}{|c|c|c|c|c|c|c|c|c|c|}
\hline ID & Name & Other Name & R.A. (J2000) & Decl. (J2000) & $z^{\mathrm{a}}$ & $E(B-V)^{\mathrm{b}}$ & $R^{\mathrm{c}}$ & $\lambda L_{\lambda}(3000 \AA)^{\mathrm{d}}$ & SampleID \\
\hline 66 & PG $1545+210$ & 3C 323.1 & $15: 47: 43.54$ & $+20: 52: 16.7$ & 0.2642 & 0.042 & 1000 & 45.52 & RLQ \\
\hline 67 & B2 $1555+33$ & & $15: 57: 29.94$ & $+33: 04: 47.0$ & 0.9420 & 0.038 & 975 & 45.02 & RLQ \\
\hline 68 & B2 $1611+34$ & DA 406 & $16: 13: 41.06$ & $+34: 12: 47.9$ & 1.3945 & 0.018 & 5825 & 44.83 & RLQ \\
\hline 69 & $3 C 334$ & & $16: 20: 21.92$ & $+17: 36: 24.0$ & 0.5553 & 0.041 & 1294 & 45.51 & RLQ \\
\hline 70 & PG $1626+554$ & & $16: 27: 56.00$ & $+55: 22: 31.0$ & 0.1317 & 0.006 & 0.10 & 45.53 & PGX \\
\hline 71 & OS 562 & & $16: 38: 13.45$ & $+57: 20: 24.0$ & 0.7506 & 0.013 & 2248 & 43.51 & RLQ \\
\hline 72 & PKS 1656+053 & & $16: 58: 33.45$ & $+05: 15: 16.4$ & 0.8890 & 0.159 & 1268 & 45.65 & RLQ \\
\hline 73 & PG 1704+608 & $3 C 351$ & $17: 04: 41.37$ & $+60: 44: 30.5$ & 0.3730 & 0.023 & 666 & 45.28 & FUSE,RLQ \\
\hline 74 & MRK 506 & & $17: 22: 39.90$ & $+30: 52: 53.0$ & 0.0428 & 0.031 & 3.11 & 44.90 & FUSE \\
\hline 75 & 4C 34.47 & B2 1721+34 & $17: 23: 20.80$ & $+34: 17: 57.9$ & 0.2055 & 0.037 & 419 & 45.81 & FUSE \\
\hline 76 & 4C 73.18 & & $19: 27: 48.49$ & $+73: 58: 01.6$ & 0.3027 & 0.133 & 1587 & 44.82 & RLQ \\
\hline 77 & MRK 509 & IRAS F20414-1054 & 20:44:09.74 & $-10: 43: 24.5$ & 0.0345 & 0.057 & 0.58 & 45.74 & FUSE \\
\hline 78 & 4C 06.69 & PKS 2145+06 & 21:48:05.46 & $+06: 57: 38.6$ & 1.0002 & 0.080 & 2102 & 45.03 & RLQ \\
\hline 79 & 4C 31.63 & B2 $2201+31 \mathrm{~A}$ & 22:03:14.97 & $+31: 45: 38.3$ & 0.2952 & 0.124 & 853 & 46.24 & RLQ \\
\hline 80 & PG 2214+139 & MRK 304 & $22: 17: 12.26$ & $+14: 14: 21.1$ & 0.0657 & 0.073 & 0.04 & 45.64 & FUSE \\
\hline 81 & PKS 2216-03 & $4 C-03.79$ & $22: 18: 52.04$ & $-03: 35: 36.9$ & 0.8993 & 0.095 & 1708 & 46.54 & RLQ \\
\hline 82 & $3 \mathrm{C} 446$ & & $22: 25: 47.26$ & $-04: 57: 01.4$ & 1.4040 & 0.075 & 21719 & 46.35 & RLQ \\
\hline 83 & 4C 11.69 & PKS 2230+11 & $22: 32: 36.41$ & $+11: 43: 50.9$ & 1.0370 & 0.072 & 5992 & 46.31 & RLQ \\
\hline 84 & PG $2251+113$ & PKS 2251+11 & $22: 54: 10.40$ & $+11: 36: 38.3$ & 0.3253 & 0.086 & 291 & 46.36 & RLQ \\
\hline 85 & PG 2349-014 & PKS 2349-10 & $23: 51: 56.13$ & $-01: 09: 13.3$ & 0.1740 & 0.027 & 556 & 45.23 & FUSE \\
\hline
\end{tabular}

Notes.

${ }^{a}$ Redshift, measured using our data (Section 4.3.1).

b From NED (http://nedwww.ipac.caltech.edu/) based on Schlegel et al. (1998).

c Radio loudness, $R=f(5 \mathrm{GHz}) / f(4215 \AA)$, calculated using our data (Section 4.3.2).

${ }^{\mathrm{d}}$ Rest-frame luminosity at $3000 \AA$, calculated using our data (Section 4.3.3).

low declination), very strong host galaxy contamination (NGC 3516), or strong variability (NGC 5548, also no simultaneous HST spectrum). Our final FUSE-HST sample includes 17 objects with quasi-simultaneous spectrophotometry extending to the FUV and covering rest wavelength from 900-9000 A. This is a heterogeneous sample with low redshift $(z<0.5)$. Shang et al. (2005) provides additional details.

\section{3. $R L Q$}

The "RLQ" sample originates with an early HST program to observe a large sample of RL quasars selected to have a small range in extended radio luminosity, a property thought to be isotropic. Limitations of $H S T$ discovered after launch required adjustments to the sample and brighter objects were substituted for fainter ones. Over the course of four cycles, HST targeted nearly 50 quasars. Quasi-simultaneous optical spectrophotometry was obtained at several observatories, primarily McDonald Observatory and KPNO. Wills et al. (1995) and Netzer et al. (1995) provide additional details of the sample. A number of the radio-core dominant quasars are blazars, with optically violent variability due to synchrotron emission from a beamed jet. We have excluded these blazars from the sample based on rapid optical variability as we regard this component as a major complication in determining intrinsic and uniform SEDs for comparison.

\subsection{Sample Properties}

In order to summarize the properties of the combined sample, we have plotted some histograms (Figure 1). We have distinguished RL and RQ quasars using radio loudness calculated with our data. We have also measured the redshift and $3000 \AA$ rest-frame continuum luminosity of the sample. Details are provided in Section 4.3.
Of the 85 objects in the final sample, there are 27 RQ and 58 RL quasars. All RQ quasars are from either PGX or FUSE-HST subsamples, having redshift less than 0.5. Most RL quasars are from the RLQ subsample and more than half of the RL quasars have redshift larger than 0.5 .

Both RL and RQ samples span about two orders of magnitude in luminosity. The RL sample has an average luminosity about six times higher. These properties reflect the original selections of the subsamples.

We emphasize that as a whole this sample is representative of $\mathrm{UV}$ /optical-bright quasars, both RL and RQ, but is not statistically complete or well matched. Particular subsamples may be appropriate for general statistical studies and comparisons only if care is taken in their selection.

\section{DATA}

We collected both photometry and spectroscopy data for this work. Many data were obtained with space telescopes, including HST, FUSE, Spitzer, Chandra, and XMM. This ensures the unprecedented quality of the SEDs. Figure 2 shows two examples of our objects, marked with wavebands and instruments used to obtain the data. These will be discussed in detail in the following sections.

Most of the data were obtained between 1991 and 2007 except for some archival radio data which were obtained much earlier (see Table 2 for details). However, the problem of AGN intrinsic variability can be neglected statistically for the SED work, especially with regard to some portions (e.g., IR) which have only very long timescale variation. Moreover, the FUV-UV-optical spectra of our sample were mostly obtained quasi-simultaneously, within weeks, specifically minimizing this problem. 

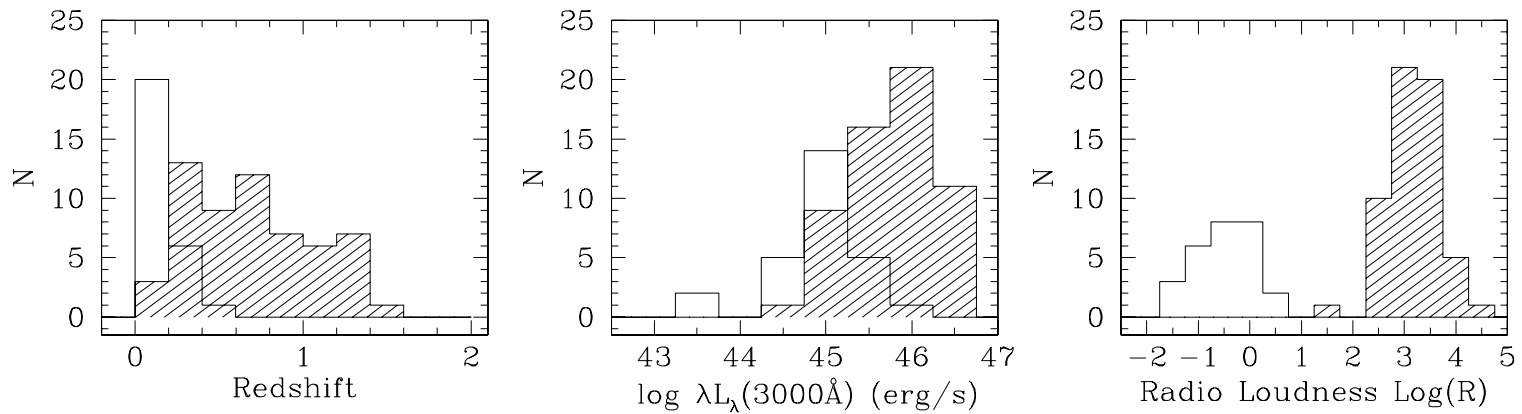

Figure 1. Distributions of sample properties for RL and RQ subsamples. Shaded bins are for RL objects.
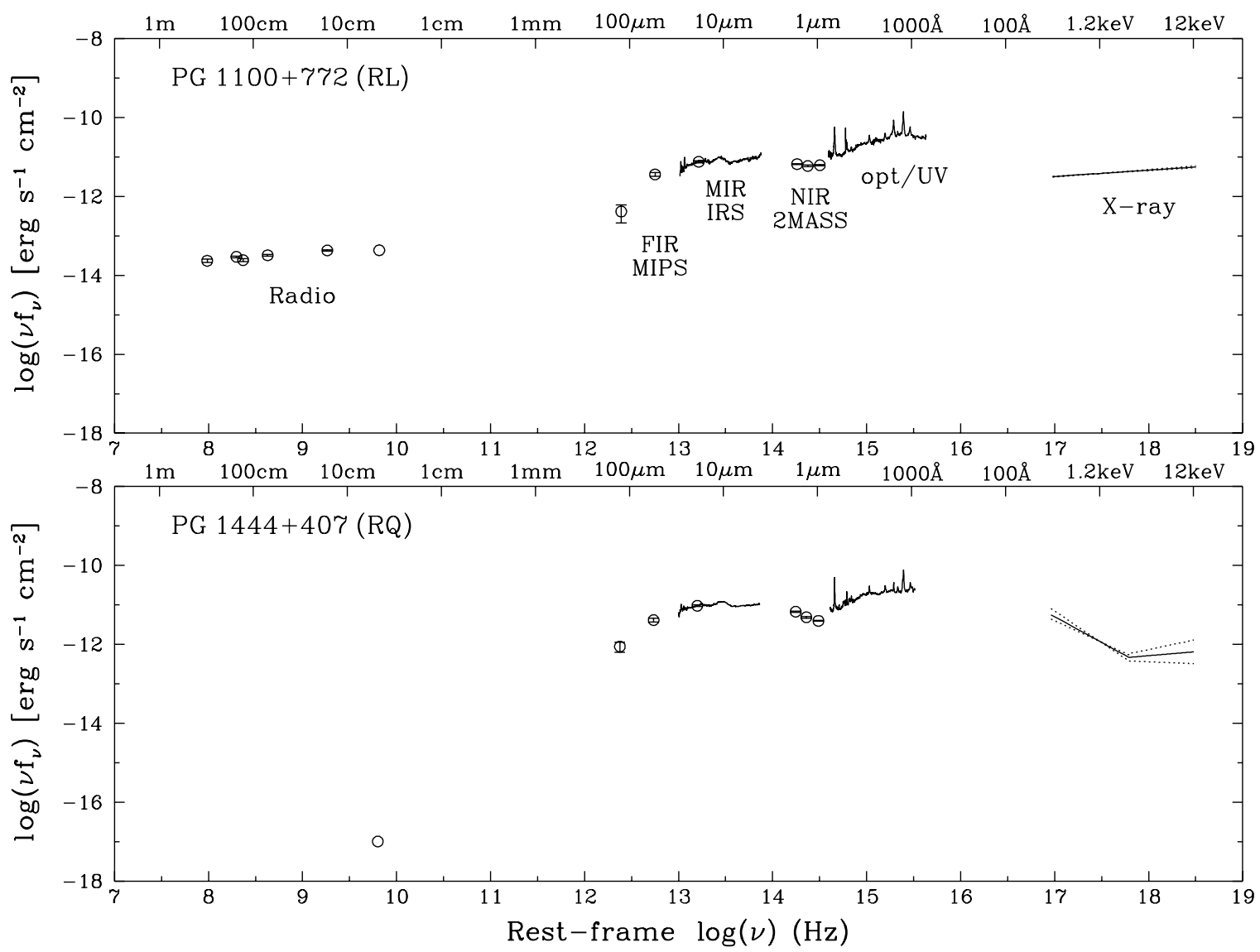

Figure 2. Examples of our collected data for two objects. Also marked are the wavebands and some instruments used to obtain the data.

Before we combine data from different bands to construct the SEDs, we applied two corrections: host galaxy correction in the near-IR (Section 4.1) and Galactic reddening in the FUV-UV-optical spectra (Section 4.2). We also derived some sample properties from the data set (Section 4.3).

\subsection{Radio}

We collect radio data for the sample from archives of highquality surveys and some data from the literature. Table 2 lists all the references. We choose the frequency range from $74 \mathrm{MHz}$ to about $15 \mathrm{GHz}$, where most objects have observations. We include surveys of similar frequencies (e.g., 325 and $365 \mathrm{MHz}$; $4850,4890,4990$, and $5000 \mathrm{MHz}$ ) in order to maximize the number of objects with available observations in a similar frequency range.

The total fluxes at each frequency are listed in Table 3 and used in the SED construction and analysis. When a survey or an observation resolves the core and lobes, we make sure to get the total flux by including all the components. In doing so, we check the positions of each component and take advantage of the higher resolution map $\left(5^{\prime \prime}\right)$ of FIRST survey for comparison. At $1400 \mathrm{MHz}$, we use the total flux density from NVSS in preference to that from FIRST for all but three objects (IRAS F07546+3928, Mrk 506, and PG 1115+407) that are not included in the NVSS catalog. NVSS has a spatial resolution comparable to other major radio surveys we use, and generally includes all the radio flux, while FIRST resolves many sources into multiple regions of emission. We did check the FIRST images and collected the measurements for each object, which, when the pieces are summed, give results consistent with those from NVSS.

Most RQ objects are not detected in the radio surveys, which can provide only an upper limit. We therefore search the literature to obtain at least one detection from individual studies. 
Table 2

Radio Surveys

\begin{tabular}{|c|c|c|c|c|}
\hline $\begin{array}{l}\text { Frequency } \\
(\mathrm{MHz})\end{array}$ & Surveys & $\begin{array}{c}\text { Number of } \\
\text { Objects Included }\end{array}$ & $\begin{array}{c}\text { Resolution } \\
\left({ }^{\prime \prime}\right)\end{array}$ & References \\
\hline 74 & VLSS & 57 & 45 & 1 \\
\hline 151 & $7 \mathrm{C}$ & 30 & 70 & 2 \\
\hline 178 & $4 \mathrm{C}$ & 44 & $1380-1860$ & 3 \\
\hline 325 & WENSS & 29 & 54 & 4 \\
\hline 365 & TEXAS & 55 & 22.1 & 5 \\
\hline 408 & MRC & 23 & 157 & 6 \\
\hline 1400 & NVSS/FIRST & $64 / 3$ & $45 / 5$ & 7 \\
\hline 1420 & Ulvestad.2005 & 2 & $<0.01$ & 8 \\
\hline 1490 & Barvainis.1996 & 4 & $<40$ & 9 \\
\hline 2270 & Ulvestad.2005 & 2 & $<0.01$ & 8 \\
\hline 4800 & Leipski.2006 & 3 & $<1.7$ & 10 \\
\hline 4850 & GB6 & 50 & 210 & 11 \\
\hline 4850 & $\mathrm{PMN}$ & 8 & 252 & 12 \\
\hline 4890 & Barvainis.1996 & 6 & $<40$ & 9 \\
\hline 4990 & Ulvestad.2005 & 2 & $<0.01$ & 8 \\
\hline 5000 & Kellermann.1989 & 21 & 0.5 & 13 \\
\hline 5000 & Gear.1994 & 2 & 714 & 14 \\
\hline 8000 & Gear.1994 & 3 & 444 & 14 \\
\hline 8480 & Barvainis.1996 & 3 & $<40$ & 9 \\
\hline 8600 & Barvainis.2005 & 1 & $\sim 1.3$ & 15 \\
\hline 10700 & Kellermann.1973 & 24 & 171 & 16 \\
\hline 14000 & Gear.1994 & 3 & 252 & 14 \\
\hline 14900 & Genzel.1976 & 12 & 59 & 17 \\
\hline 14900 & Barvainis.1996 & 6 & $<40$ & 9 \\
\hline 15200 & Bolton.2004 & 1 & $<252$ & 18 \\
\hline
\end{tabular}

Notes. Radio surveys and references used to collect SED data.

References. (1) The VLA Low-Frequency Sky Survey (Cohen et al. 2007); (2) 7C $151 \mathrm{MHz}$ Survey (Hales et al. 2007); (3) 4C Survey (Pilkington \& Scott 1965; Gower et al. 1967); (4) The Westerbork Northern Sky Survey (Rengelink et al. 1997; de Bruyn et al. 1998); (5) The Texas Survey of Radio Sources (Douglas et al. 1996); (6) The Molonglo Reference Catalogue of Radio Sources (Large et al. 1981, 1991); (7) The NRAO VLA Sky Survey (Condon et al. 1998); Faint Images of the Radio Sky at Twenty-Centimeters (Becker et al. 1995); (8) Ulvestad et al. 2005; (9) Barvainis et al. 1996; (10) Leipski et al. 2006; (11) GB6 (12) The Parkes-MIT-NRAO (Griffith et al. 1995); (13) Kellermann et al. 1989; (14) Gear et al. 1994; (15) Barvainis et al. 2005; (16) Kellermann \& Pauliny-Toth 1973; (17) Genzel et al. 1976; (18) Bolton et al. 2004.

Table 3

Radio Fluxes

\begin{tabular}{|c|c|c|c|c|c|}
\hline ID & Object & $\begin{array}{c}v \\
(\mathrm{MHz})\end{array}$ & $\begin{array}{c}f_{v} \\
(\mathrm{mJy})\end{array}$ & $\begin{array}{c}\Delta f_{v} \\
(\mathrm{mJy})\end{array}$ & Reference \\
\hline \multirow[t]{4}{*}{1} & MC2 0042+101 & 74 & 3380 & 390 & VLSS \\
\hline & & 365 & 540 & 47 & TEXAS \\
\hline & & 1400 & 218.8 & 7.0 & NVSS \\
\hline & & 4850 & 83 & 8.1 & GB6 \\
\hline \multirow[t]{3}{*}{2} & PG $0052+251$ & 4800 & 0.61 & 0.03 & Leipski et al. (2006) \\
\hline & & 5000 & 0.74 & & Kellermann et al. (1989) \\
\hline & & 8600 & 0.7 & & Barvainis et al. (2005) \\
\hline \multirow[t]{5}{*}{3} & PKS 0112-017 & 74 & 780 & 120 & VLSS \\
\hline & & 365 & 974 & 25 & TEXAS \\
\hline & & 408 & 1110 & 70 & MRC \\
\hline & & 1400 & 1076.2 & 32.3 & NVSS \\
\hline & & 4850 & 1437 & 75 & PMN \\
\hline
\end{tabular}

(This table is available in its entirety in a machine-readable form in the online journal. A portion is shown here for guidance regarding its form and content.)

\subsection{Far-IR}

Far-IR photometry at 24, 70, and $160 \mu \mathrm{m}$ from Multiband Imaging Photometer for Spitzer (MIPS; Rieke et al. 2004) is
Table 4 Spitzer MIPS FIR Fluxes

\begin{tabular}{|c|c|c|c|c|}
\hline \multirow[t]{2}{*}{ ID } & \multirow[t]{2}{*}{ Object } & \multicolumn{3}{|c|}{ Flux (mJy) } \\
\hline & & $24 \mu \mathrm{m}$ & $70 \mu \mathrm{m}$ & $160 \mu \mathrm{m}$ \\
\hline 2 & PG $0052+251$ & $70 . \pm 3.4$ & $76.1 \pm 9.1$ & $65.1 \pm 14$ \\
\hline 5 & $3 \mathrm{C} 47$ & $33.5 \pm 2.35$ & $31 . \pm 5.14$ & $15.2 \pm 7.58$ \\
\hline 10 & $3 \mathrm{C} 175$ & $12.3 \pm 1.43$ & $25.3 \pm 5.06$ & $<2.5$ \\
\hline 11 & $3 \mathrm{C} 186$ & $6.79 \pm 1.06$ & . & . \\
\hline 13 & IRAS F07546+3928 & $220 . \pm 6.03$ & 154. \pm 11.5 & $<1.08$ \\
\hline 14 & 3C 207 & $11.9 \pm 1.4$ & $23.7 \pm 4.56$ & $57.8 \pm 13.1$ \\
\hline 15 & PG 0844+349 & $92.4 \pm 3.91$ & $63.2 \pm 7.93$ & 107. \pm 18.1 \\
\hline 20 & PG 0947+396 & $49.8 \pm 2.87$ & 117. \pm 10.2 & 111. \pm 18.3 \\
\hline 21 & PG $0953+414$ & $42 . \pm 2.64$ & 18. \pm 5.81 & $16.6 \pm 7.12$ \\
\hline 24 & PG $1001+054$ & $35.9 \pm 2.44$ & $36.4 \pm 6.09$ & $<0.128$ \\
\hline 28 & PG $1100+772$ & 46. \pm 2.76 & $63.9 \pm 7.07$ & $16.8 \pm 8.21$ \\
\hline 29 & PG 1103-006 & $32.5 \pm 2.32$ & $21.9 \pm 5.52$ & $17.6 \pm 7.58$ \\
\hline 30 & $3 C 254$ & $12.2 \pm 1.42$ & $11.7 \pm 3.18$ & $11.6 \pm 6.11$ \\
\hline 31 & PG 1114+445 & $133 . \pm 4.69$ & $50.3 \pm 6.85$ & $<1.15$ \\
\hline 32 & PG 1115+407 & 44. \pm 2.7 & $175 . \pm 11.9$ & 143. \pm 20.6 \\
\hline 33 & PG $1116+215$ & $102 . \pm 4.11$ & $63.1 \pm 8.43$ & $30.5 \pm 9.69$ \\
\hline 36 & $3 \mathrm{C} 263$ & $28.6 \pm 2.18$ & $50.8 \pm 7.02$ & $18.1 \pm 7.44$ \\
\hline 40 & PG $1202+281$ & $81.2 \pm 3.67$ & 112. \pm 10.1 & $32.8 \pm 10.2$ \\
\hline 42 & PG 1216+069 & $24.2 \pm 2.01$ & $15.4 \pm 4.66$ & $<0.845$ \\
\hline 43 & PG $1226+023$ & $511 . \pm 9.2$ & 488. \pm 20.2 & 299. \pm 29.8 \\
\hline 45 & $3 \mathrm{C} 277.1$ & $20.5 \pm 1.85$ & $21.4 \pm 4.12$ & $<1.29$ \\
\hline 46 & PG $1259+593$ & $21.2 \pm 1.87$ & $10.7 \pm 3.7$ & $9.03 \pm 5.51$ \\
\hline 48 & PG $1309+355$ & $104 . \pm 4.15$ & $85.8 \pm 8.86$ & $45.9 \pm 11.7$ \\
\hline 49 & PG $1322+659$ & $43.3 \pm 2.68$ & $113 . \pm 10$ & $92.3 \pm 16.7$ \\
\hline 50 & 3C 288.1 & $8.32 \pm 1.17$ & $\ldots$ & \\
\hline 51 & PG 1351+640 & $423 . \pm 8.36$ & 567. \pm 21.7 & 187. \pm 23.6 \\
\hline 53 & PG $1352+183$ & $31.6 \pm 2.29$ & $10.1 \pm 4.07$ & $<0.164$ \\
\hline 56 & PG $1402+261$ & $100 . \pm 4.08$ & 263. \pm 14.8 & $86.6 \pm 16.2$ \\
\hline 57 & PG $1411+442$ & $122 . \pm 4.48$ & $101 . \pm 9.04$ & $70.4 \pm 14.5$ \\
\hline 58 & PG $1415+451$ & $59.3 \pm 3.13$ & $96.6 \pm 8.87$ & $54.4 \pm 12.7$ \\
\hline 59 & PG $1425+267$ & $42.7 \pm 2.66$ & $75.8 \pm 8.25$ & $14.9 \pm 6.86$ \\
\hline 60 & PG $1427+480$ & $41.9 \pm 2.63$ & $91.7 \pm 9.4$ & $<1.06$ \\
\hline 61 & PG $1440+356$ & 177. \pm 5.42 & 738. \pm 24.5 & 438. \pm 36 \\
\hline 62 & PG $1444+407$ & $60 . \pm 3.15$ & $75.6 \pm 8.53$ & 37. \pm 10.6 \\
\hline 63 & PG $1512+370$ & $31.4 \pm 2.28$ & $37.3 \pm 5.77$ & $<0.999$ \\
\hline 64 & PG $1534+580$ & 173. \pm 5.34 & 172. \pm 11.6 & $60.4 \pm 13.4$ \\
\hline 65 & PG 1543+489 & $101 . \pm 4.09$ & $285 . \pm 15$ & $82.8 \pm 15.7$ \\
\hline 66 & PG $1545+210$ & $33.8 \pm 2.37$ & $19.4 \pm 3.96$ & $<0.528$ \\
\hline 67 & B2 $1555+33$ & $3.44 \pm 0.757$ & $\cdots$ & $\ldots$ \\
\hline 69 & $3 \mathrm{C} 334$ & $35.2 \pm 2.41$ & $86.9 \pm 8.58$ & $18.4 \pm 7.39$ \\
\hline 70 & PG $1626+554$ & $16.5 \pm 1.65$ & $16.3 \pm 4.38$ & $4.83 \pm 3.8$ \\
\hline 73 & PG 1704+608 & $105 . \pm 4.16$ & 188. \pm 12.2 & $41.6 \pm 11.3$ \\
\hline 74 & MRK 506 & $82.6 \pm 3.69$ & 196. \pm 12.7 & $292 . \pm 29.4$ \\
\hline 75 & 4C 34.47 & $55.2 \pm 3.02$ & $27.1 \pm 4.73$ & $<0.326$ \\
\hline 77 & MRK 509 & $602 . \pm 9.97$ & $1440 . \pm 34.4$ & $689 . \pm 45.2$ \\
\hline 79 & 4C 31.63 & $70.4 \pm 3.41$ & $79.7 \pm 8.24$ & $33.7 \pm 10$ \\
\hline 80 & PG 2214+139 & $94.8 \pm 3.96$ & $79.6 \pm 8.66$ & $48.1 \pm 12$ \\
\hline 83 & 4C 11.69 & $33.4 \pm 2.35$ & 138. \pm 10.6 & $150 . \pm 21.1$ \\
\hline 84 & PG 2251+113 & $43.8 \pm 2.69$ & $59.7 \pm 7.46$ & $<1.21$ \\
\hline 85 & PG 2349-014 & $72 . \pm 3.45$ & $155 . \pm 11.8$ & $88.2 \pm 16.2$ \\
\hline
\end{tabular}

Notes. The upper limits for $160 \mu \mathrm{m}$ are $3 \sigma$ limits, where $\sigma$ is the standard deviation of the local sky background.

available for 50 objects in our sample (Table 4). In addition to archive data, we obtained new data explicitly for this study. All these observations were made with MIPS photometry mode.

We perform aperture photometry on each object (point source) in all three bands and apply corresponding aperture corrections based on the aperture radius and sky annulus sizes listed in the MIPS Instrument Handbook. Although the MIPS handbook quotes a $10 \%$ flux calibration uncertainty for bright 


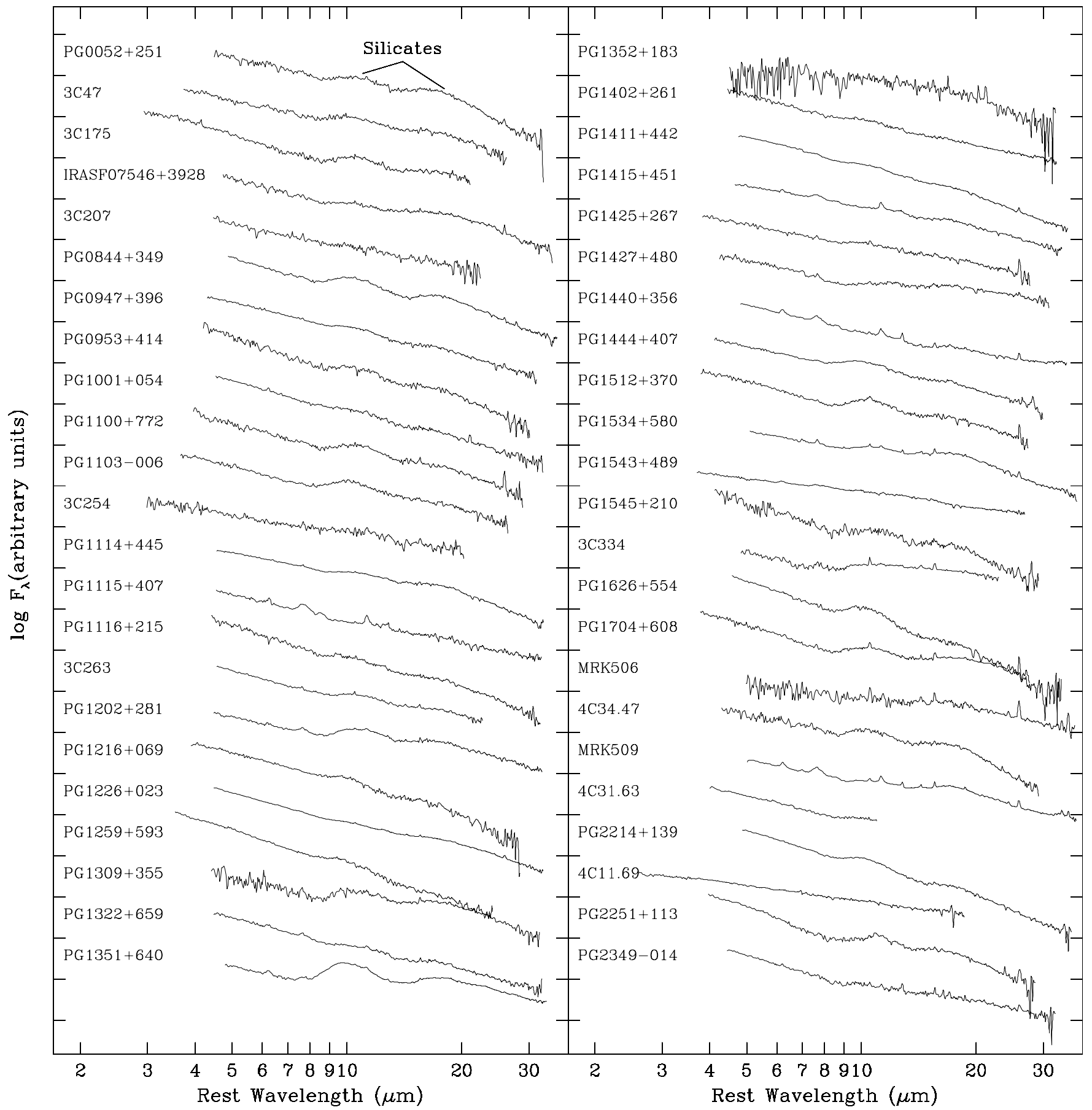

Figure 3. Available mid-infrared spectra from Spitzer IRS for our sample. The bumps around 10 and $18 \mu \mathrm{m}$ are silicate features.

sources, the photometry uncertainties can be up to $16 \%$ for $24 \mu \mathrm{m}, 40 \%$ for $70 \mu \mathrm{m}$, and $60 \%$ for $160 \mu \mathrm{m}$ for the faint objects in our sample. Moreover, several objects are not detected at $160 \mu \mathrm{m}$ and we estimated a $3 \sigma$ upper limit for them, where $\sigma$ is the standard deviation of the sky background around the source position.

\subsection{Mid-IR}

The mid-IR spectroscopy from the Spitzer Infrared Spectrograph (IRS; Houck et al. 2004; Werner et al. 2004) were obtained for 46 objects from both archival observations and new observations made for this study. We use data from four low-resolution modules covering observed wavelengths from 5 to $40 \mu \mathrm{m}$.
Since all our objects are essentially point sources, we obtained the spectra from standard post-basic calibrated data (PBCD) products. Before we combined individual segments from different wavebands, we removed flagged data points and obvious spurious points at the edges of detectors. The final spectra are shown in Figure 3, covering rest wavelength from about $\sim 3-35 \mu \mathrm{m}$ for the redshifts of our sample. The spectrophotometric calibration uncertainty is within $15 \%$, and this is also verified with our $24 \mu \mathrm{m}$ photometry of MIPS.

MIR spectra show clear broad silicate emission features around 10 and $18 \mu \mathrm{m}$, and narrow emission lines such as [S IV] $\lambda 10.5 \mu \mathrm{m},[\mathrm{Ne} \mathrm{v}] \lambda 14.3 \mu \mathrm{m},[\mathrm{Ne}$ III] $\lambda 15.6 \mu \mathrm{m}$, and $[\mathrm{O}$ IV $] \lambda 25.9 \mu \mathrm{m}$. These features have been investigated in detail 
in previous studies (e.g., Hao et al. 2005; Weedman et al. 2005; Dale et al. 2009; Goulding \& Alexander 2009; Diamond-Stanic et al. 2009; Pereira-Santaella et al. 2010; Tommasin et al. 2010), and have been kept in our analyses.

\subsection{Near-IR}

We rely on 2MASS photometry in the near-IR (Skrutskie et al. 2006), supplemented by our own observations with NASA's IRTF $^{14}$ and the HST. The 2MASS point-source catalog has 79 members of our sample. Table 5 provides magnitudes derived from both point-source profile fitting and aperture photometry from 2MASS, along with a flag (ext) that indicates the source is also listed in the 2MASS extended source catalog and then the aperture magnitudes are obtained from there instead. For objects only in the 2MASS point-source catalog, the apertures are $4^{\prime \prime}$ in radius, while for objects in the extended source catalog the apertures are $14^{\prime \prime}$ in radius.

In the absence of additional information, we use the 2MASS point-source profile fitting magnitudes as the AGN magnitudes, but in many cases we can do better than this. Using host galaxy measurements, we estimated and subtracted the host galaxy contribution to obtain AGN magnitudes as described in Section 4.1.

\subsection{Near-UV-Optical}

We have UV-optical spectrophotometry for all the objects from our previous studies (Wills et al. 1995; Netzer et al. 1995; Shang et al. 2003, 2005, 2007). We follow the general observing and data reduction procedures to obtain the spectra. We give a brief summary here.

The optical spectra were obtained from ground-based telescopes in long-slit mode. They were re-analyzed in a consistent way for all the three subsamples. The host galaxy contribution was checked carefully and removed as much as possible when extracting the spectra. The host contribution can only significantly affect the red part of the optical spectra and we used different aperture sizes to verify that the host galaxy contamination in the final spectra is undetectable. For several higher redshift objects in the RLQ sample, we also obtained near-IR spectra from UKIRT to cover the important rest-frame $\mathrm{H} \alpha$ regions.

The near-UV spectra are from HST Faint Object Spectrograph (FOS) for the RLQ and PGX subsamples over several cycles. For the FUSE-HST sample, the spectra are from Space Telescope Imaging Spectrograph (STIS) Snap programs (Kriss 2000). Most of these UV spectra were obtained quasi-simultaneously (within weeks) with our optical spectra to reduce the uncertainty caused by their intrinsic variability. The standard flux calibration is very good and is usually consistent with our optical data (Shang et al. 2005, 2007). The typical flux density uncertainty is less than $5 \%$.

\subsection{Far-UV}

While the FUV portion of the SED is relatively narrow, it is of great interest for several reasons. The turnover and energy peak of the optical-UV "big blue bump" is in or near the FUV. This portion of the SED is the part we can observe in most quasars that is closest to the peak of the ionizing continuum.

\footnotetext{
14 The Infrared Telescope Facility is operated by the University of Hawaii under Cooperative Agreement No. NNX-08AE38A with the National

Aeronautics and Space Administration, Science Mission Directorate, Planetary Astronomy Program.
}

The ionizing continuum powers emission lines that have been used to estimate black hole masses and probably also drives high-velocity outflows that interact with the environment.

We provide FUV data when available. High-resolution observed-frame FUV spectra, from $905-1187 \AA$, is available from FUSE (Moos et al. 2000) for a fraction of our sample, primarily the FUSE-HST subsample (17 objects). Shang et al. (2005) provide details about the FUSE data of this subsample and cite additional related technical material about FUSE.

There was also a FUSE program specifically targeting the PGX sample but most of these objects turned out to be too faint for FUSE. In addition to the FUSE-HST sample, the FUSE archive does have observations of several additional quasars in our sample, good enough for SED purposes: 3C 263, PG $1116+215$, PG 1216+069, PG 1402+261, PG 1415+451, PG $1440+356$, PG $1626+554$, and these data are included in the same way as in Shang et al. (2005). All 24 objects with FUSE data and their rest-frame wavelength coverage in FUSE are listed in Table 8.

Our sample with FUSE data will be biased to lower luminosity, lower redshift objects, typically bright Seyfert 1 galaxies. Higher redshift quasars will fortunately have the rest-frame FUV redshifted to longer wavelengths observable with $H S T$, so this bias is not very significant.

$$
\text { 3.7. X-Ray }
$$

The X-ray data are collected from Chandra, XMM, and ROSAT sources reported in the literature. Because of their higher sensitivity and broader energy coverage, we always choose Chandra and XMM data when available; otherwise, we resort to ROSAT. We have a total of 71 objects with X-ray information, 34 from ROSAT data.

For individual X-ray studies of AGNs, the data are usually fitted with different models and components to reveal detailed $\mathrm{X}$-ray properties. Sometimes the models are very complicated, but for the purpose of SED work, we focus on the overall shape of the energy distribution in this region, therefore, we try to choose the simplest, best-fitting models. This includes either a single power law or a broken power law. In addition to individual studies, we also obtained the results of three objects from the Chandra Source Catalog (CSC; Evans et al. 2010).

The spectral indices and flux densities from different studies have been converted to a uniform system for consistency, $f_{v}=f_{0} E^{\alpha}$, where $f_{0}$ is the flux density at $1 \mathrm{keV}$, in units of erg $\mathrm{s}^{-1} \mathrm{~cm}^{-2} \mathrm{~Hz}^{-1}, E$ in $\mathrm{keV}$, and $\alpha$ is the power-law (or broken power-law) spectral index. The results are listed in Table 9 along with the references.

In order to obtain the SED in the X-ray domain, we rebuild the power-law or broken power-law "spectra" using the spectral index $\alpha$ and $f_{0}$ within the instrument-related energy ranges in the observed frame. A sampling of $0.1 \mathrm{keV}$ is enough to show the X-ray SED shape. We also use the errors of $\alpha$ to estimate the uncertainty of the X-ray SEDs.

\section{CORRECTIONS AND MEASUREMENTS}

\subsection{Near-infrared Host Galaxy Corrections}

Most of our objects are UV-optical bright quasars and the host galaxy contamination to the AGN light is not large. When possible, we have tried to estimate the host galaxy contribution using photometry at $H$-band, where, due to typical SED shapes of galaxies and quasars, the fraction of host galaxy contribution may be maximized in contrast to AGN light at redshifts around 
Table 5

Near-IR $J H K$ Magnitudes from 2MASS

\begin{tabular}{|c|c|c|c|c|c|c|c|c|}
\hline \multirow[t]{2}{*}{ ID } & \multirow[t]{2}{*}{ Object } & \multicolumn{3}{|c|}{ Profile Fitting Magnitude } & \multicolumn{3}{|c|}{ Aperture Magnitude } & \multirow[t]{2}{*}{$\overline{E x t^{a}}$} \\
\hline & & $J$ & $H$ & $K$ & $J$ & $H$ & $K$ & \\
\hline 2 & PG $0052+251$ & $13.891 \pm 0.032$ & $13.174 \pm 0.032$ & $12.239 \pm 0.022$ & $13.813 \pm 0.022$ & $13.071 \pm 0.018$ & $12.194 \pm 0.023$ & \\
\hline 3 & PKS 0112-017 & $16.935 \pm 0.172$ & $15.810 \pm 0.131$ & $15.664 \pm 0.212$ & $16.311 \pm 0.183$ & $15.654 \pm 0.269$ & $15.087 \pm 0.321$ & \\
\hline 4 & $3 \mathrm{C} 37$ & $16.734 \pm 0.154$ & 15.913 & $15.099 \pm 0.136$ & $16.528 \pm 0.400$ & & $15.248 \pm 0.281$ & \\
\hline 5 & $3 \mathrm{C} 47$ & $16.352 \pm 0.112$ & $15.445 \pm 0.111$ & $14.553 \pm 0.093$ & $16.086 \pm 0.212$ & $15.248 \pm 0.219$ & $14.339 \pm 0.093$ & \\
\hline 6 & 4C 01.04 & $15.835 \pm 0.083$ & $14.866 \pm 0.082$ & $13.822 \pm 0.058$ & $15.204 \pm 0.154$ & $14.459 \pm 0.105$ & $13.373 \pm 0.138$ & ext \\
\hline 7 & $4 \mathrm{C} 10.06$ & $15.351 \pm 0.051$ & $14.547 \pm 0.049$ & $13.796 \pm 0.050$ & $15.279 \pm 0.061$ & $14.572 \pm 0.103$ & $13.775 \pm 0.086$ & \\
\hline 8 & PKS 0403-13 & $15.613 \pm 0.062$ & $14.822 \pm 0.081$ & $14.122 \pm 0.065$ & $15.534 \pm 0.118$ & $14.186 \pm 0.155$ & $13.938 \pm 0.226$ & ext \\
\hline 9 & $3 \mathrm{C} 110$ & $14.875 \pm 0.036$ & $14.733 \pm 0.050$ & $13.937 \pm 0.048$ & $14.875 \pm 0.059$ & $14.853 \pm 0.133$ & $13.902 \pm 0.170$ & \\
\hline 10 & $3 \mathrm{C} 175$ & $14.882 \pm 0.069$ & $14.677 \pm 0.080$ & $14.002 \pm 0.064$ & $14.742 \pm 0.093$ & $14.423 \pm 0.067$ & $13.935 \pm 0.147$ & \\
\hline 12 & B2 $0742+31$ & $14.478 \pm 0.031$ & $13.780 \pm 0.033$ & $12.950 \pm 0.030$ & $14.453 \pm 0.020$ & $13.719 \pm 0.045$ & $12.900 \pm 0.030$ & \\
\hline 13 & IRAS F07546+3928 & $12.909 \pm 0.024$ & $12.014 \pm 0.031$ & $11.013 \pm 0.021$ & $12.885 \pm 0.016$ & $11.971 \pm 0.021$ & $10.998 \pm 0.027$ & \\
\hline 14 & 3C 207 & $16.686 \pm 0.158$ & $15.961 \pm 0.141$ & $15.037 \pm 0.117$ & $16.253 \pm 0.169$ & $16.241 \pm 0.255$ & $14.782 \pm 0.168$ & \\
\hline 15 & PG 0844+349 & $13.409 \pm 0.028$ & $12.784 \pm 0.029$ & $12.015 \pm 0.024$ & $13.328 \pm 0.013$ & $12.669 \pm 0.012$ & $11.958 \pm 0.013$ & \\
\hline 16 & PKS 0859-14 & $15.725 \pm 0.076$ & $14.849 \pm 0.063$ & $14.781 \pm 0.120$ & $15.687 \pm 0.125$ & $15.026 \pm 0.200$ & $14.936 \pm 0.174$ & \\
\hline 17 & $3 \mathrm{C} 215$ & $16.860 \pm 0.159$ & $15.845 \pm 0.173$ & $15.049 \pm 0.100$ & $16.666 \pm 0.347$ & $15.738 \pm 0.347$ & $14.752 \pm 0.096$ & \\
\hline 18 & 4C 39.25 & $15.342 \pm 0.051$ & $14.852 \pm 0.072$ & $14.002 \pm 0.055$ & $15.246 \pm 0.087$ & $15.020 \pm 0.135$ & $13.907 \pm 0.049$ & \\
\hline 19 & $4 \mathrm{C} 40.24$ & $16.708 \pm 0.160$ & $16.186 \pm 0.244$ & $15.383 \pm 0.199$ & $16.972 \pm 0.078$ & $18.096 \pm 4.651$ & $15.636 \pm 0.347$ & \\
\hline 20 & PG 0947+396 & $14.775 \pm 0.038$ & $13.943 \pm 0.036$ & $12.767 \pm 0.028$ & $14.415 \pm 0.071$ & $13.716 \pm 0.082$ & $12.635 \pm 0.062$ & ext \\
\hline 21 & PG $0953+414$ & $14.196 \pm 0.028$ & $13.483 \pm 0.027$ & $12.532 \pm 0.025$ & $14.182 \pm 0.032$ & $13.455 \pm 0.050$ & $12.509 \pm 0.035$ & \\
\hline 22 & 4C 55.17 & $15.676 \pm 0.069$ & $14.944 \pm 0.075$ & $14.190 \pm 0.065$ & $15.816 \pm 0.034$ & $14.772 \pm 0.077$ & $14.099 \pm 0.099$ & \\
\hline 23 & $3 \mathrm{C} 232$ & $14.945 \pm 0.039$ & $14.443 \pm 0.050$ & $13.760 \pm 0.042$ & $14.905 \pm 0.049$ & $14.478 \pm 0.023$ & $13.810 \pm 0.060$ & \\
\hline 24 & PG $1001+054$ & $15.056 \pm 0.051$ & $14.191 \pm 0.039$ & $13.105 \pm 0.034$ & $14.988 \pm 0.053$ & $14.176 \pm 0.072$ & $13.031 \pm 0.035$ & \\
\hline 25 & $4 \mathrm{C} 22.26$ & $16.776 \pm 0.155$ & 16.575 & $15.648 \pm 0.178$ & $16.937 \pm 0.277$ & $\ldots$ & $15.877 \pm 0.332$ & \\
\hline 26 & $4 C 41.21$ & $15.364 \pm 0.057$ & $14.701 \pm 0.068$ & $13.964 \pm 0.059$ & $15.344 \pm 0.083$ & $14.738 \pm 0.274$ & $13.880 \pm 0.048$ & \\
\hline 27 & 4C 20.24 & $16.031 \pm 0.071$ & $15.245 \pm 0.080$ & $14.611 \pm 0.073$ & $15.822 \pm 0.118$ & $15.304 \pm 0.223$ & $14.475 \pm 0.163$ & \\
\hline 28 & PG $1100+772$ & $14.471 \pm 0.034$ & $13.928 \pm 0.048$ & $13.053 \pm 0.036$ & $14.448 \pm 0.041$ & $13.752 \pm 0.049$ & $12.960 \pm 0.026$ & \\
\hline 29 & PG $1103-006$ & $15.313 \pm 0.050$ & $14.787 \pm 0.062$ & $13.855 \pm 0.061$ & $15.343 \pm 0.080$ & $14.652 \pm 0.088$ & $13.763 \pm 0.065$ & \\
\hline 30 & $3 \mathrm{C} 254$ & $15.879 \pm 0.084$ & $15.293 \pm 0.105$ & $14.673 \pm 0.109$ & $15.389 \pm 0.194$ & $14.468 \pm 0.180$ & $13.997 \pm 0.228$ & ext \\
\hline 31 & PG $1114+445$ & $14.184 \pm 0.028$ & $13.529 \pm 0.029$ & $12.335 \pm 0.021$ & $14.150 \pm 0.021$ & $13.432 \pm 0.056$ & $12.292 \pm 0.021$ & \\
\hline 32 & PG $1115+407$ & $14.660 \pm 0.056$ & $13.798 \pm 0.058$ & $12.763 \pm 0.036$ & $14.463 \pm 0.029$ & $13.583 \pm 0.025$ & $12.625 \pm 0.027$ & \\
\hline 33 & PG $1116+215$ & $13.592 \pm 0.027$ & $12.684 \pm 0.026$ & $11.540 \pm 0.020$ & $13.550 \pm 0.014$ & $12.655 \pm 0.024$ & $11.513 \pm 0.015$ & \\
\hline 35 & PKS $1127-14$ & $15.276 \pm 0.060$ & $14.425 \pm 0.047$ & $13.594 \pm 0.054$ & $15.325 \pm 0.046$ & $14.312 \pm 0.047$ & $13.577 \pm 0.042$ & \\
\hline 36 & $3 C 263$ & $14.826 \pm 0.039$ & $14.392 \pm 0.044$ & $13.655 \pm 0.043$ & $14.753 \pm 0.058$ & $14.362 \pm 0.132$ & $13.618 \pm 0.061$ & \\
\hline 37 & MC2 1146+111 & $16.572 \pm 0.148$ & $16.071 \pm 0.206$ & $15.596 \pm 0.250$ & $16.437 \pm 0.233$ & $15.390 \pm 0.207$ & $15.478 \pm 0.369$ & \\
\hline 38 & $4 C 49.22$ & $15.803 \pm 0.063$ & $14.952 \pm 0.078$ & $13.812 \pm 0.043$ & $15.438 \pm 0.182$ & $14.527 \pm 0.212$ & $13.489 \pm 0.126$ & ext \\
\hline 39 & TEX $1156+213$ & $15.711 \pm 0.063$ & $14.915 \pm 0.074$ & $13.935 \pm 0.059$ & $15.581 \pm 0.078$ & $14.859 \pm 0.071$ & $13.823 \pm 0.111$ & \\
\hline 40 & PG $1202+281$ & $14.754 \pm 0.037$ & $14.030 \pm 0.039$ & $12.878 \pm 0.028$ & $14.623 \pm 0.042$ & $13.859 \pm 0.040$ & $12.857 \pm 0.034$ & \\
\hline 41 & $4 \mathrm{C} 64.15$ & $16.735 \pm 0.161$ & $15.801 \pm 0.183$ & $15.458 \pm 0.169$ & $16.793 \pm 0.289$ & $16.224 \pm 0.470$ & $15.512 \pm 0.244$ & \\
\hline 42 & PG 1216+069 & $14.603 \pm 0.052$ & $13.974 \pm 0.051$ & $13.309 \pm 0.035$ & $14.514 \pm 0.056$ & $13.915 \pm 0.098$ & $13.427 \pm 0.040$ & \\
\hline 43 & PG $1226+023$ & $11.766 \pm 0.027$ & $11.047 \pm 0.027$ & $9.976 \pm 0.023$ & $11.692 \pm 0.023$ & $10.953 \pm 0.023$ & $9.937 \pm 0.020$ & ext \\
\hline 45 & $3 \mathrm{C} 277.1$ & $16.478 \pm 0.103$ & $16.146 \pm 0.176$ & $14.983 \pm 0.126$ & $16.431 \pm 0.121$ & $15.895 \pm 0.252$ & $14.931 \pm 0.075$ & \\
\hline 46 & PG $1259+593$ & $14.788 \pm 0.037$ & $13.978 \pm 0.045$ & $13.072 \pm 0.033$ & $14.767 \pm 0.068$ & $13.878 \pm 0.050$ & $13.044 \pm 0.023$ & \\
\hline 47 & $3 \mathrm{C} 281$ & $16.374 \pm 0.151$ & $15.918 \pm 0.200$ & $15.093 \pm 0.199$ & $16.098 \pm 0.256$ & $15.391 \pm 0.268$ & $15.142 \pm 0.455$ & \\
\hline 48 & PG $1309+355$ & $14.199 \pm 0.030$ & $13.626 \pm 0.037$ & $12.659 \pm 0.029$ & $14.085 \pm 0.013$ & $13.501 \pm 0.013$ & $12.586 \pm 0.016$ & \\
\hline 49 & PG $1322+659$ & $14.835 \pm 0.048$ & $13.991 \pm 0.043$ & $12.850 \pm 0.032$ & $14.705 \pm 0.043$ & $13.872 \pm 0.105$ & $12.792 \pm 0.033$ & \\
\hline 50 & $3 \mathrm{C} 288.1$ & $16.309 \pm 0.109$ & $16.348 \pm 0.211$ & $15.516 \pm 0.168$ & $16.072 \pm 0.207$ & $17.493 \pm 0.854$ & $15.238 \pm 0.226$ & \\
\hline 51 & PG $1351+640$ & $13.490 \pm 0.028$ & $12.854 \pm 0.039$ & $11.869 \pm 0.025$ & $13.412 \pm 0.009$ & $12.754 \pm 0.025$ & $11.835 \pm 0.014$ & \\
\hline 53 & PG $1352+183$ & $15.004 \pm 0.051$ & $14.231 \pm 0.049$ & $13.142 \pm 0.033$ & $14.878 \pm 0.076$ & $14.184 \pm 0.024$ & $13.085 \pm 0.038$ & \\
\hline 54 & 4C 19.44 & $14.865 \pm 0.035$ & $14.687 \pm 0.081$ & $13.894 \pm 0.051$ & $14.940 \pm 0.030$ & $14.506 \pm 0.111$ & $13.889 \pm 0.083$ & \\
\hline 55 & 4C 58.29 & $15.952 \pm 0.079$ & $15.048 \pm 0.077$ & $15.058 \pm 0.137$ & $15.991 \pm 0.102$ & $14.888 \pm 0.215$ & $14.861 \pm 0.283$ & \\
\hline 56 & PG $1402+261$ & $14.410 \pm 0.036$ & $13.379 \pm 0.027$ & $12.168 \pm 0.024$ & $14.305 \pm 0.031$ & $13.370 \pm 0.035$ & $12.113 \pm 0.022$ & \\
\hline 57 & PG $1411+442$ & $13.301 \pm 0.025$ & $12.497 \pm 0.023$ & $11.505 \pm 0.021$ & $13.249 \pm 0.013$ & $12.461 \pm 0.012$ & $11.474 \pm 0.012$ & \\
\hline 58 & PG $1415+451$ & $14.073 \pm 0.029$ & $13.170 \pm 0.030$ & $12.237 \pm 0.026$ & $13.979 \pm 0.029$ & $13.038 \pm 0.030$ & $12.193 \pm 0.030$ & \\
\hline 59 & PG $1425+267$ & $15.188 \pm 0.057$ & $14.437 \pm 0.062$ & $13.577 \pm 0.047$ & $15.113 \pm 0.084$ & $14.304 \pm 0.088$ & $13.543 \pm 0.048$ & \\
\hline 60 & PG $1427+480$ & $15.212 \pm 0.051$ & $14.475 \pm 0.060$ & $13.482 \pm 0.041$ & $15.096 \pm 0.081$ & $14.624 \pm 0.093$ & $13.434 \pm 0.067$ & \\
\hline 61 & PG $1440+356$ & $12.936 \pm 0.025$ & $12.001 \pm 0.024$ & $11.060 \pm 0.019$ & $12.904 \pm 0.007$ & $11.982 \pm 0.011$ & $11.061 \pm 0.007$ & \\
\hline 62 & PG $1444+407$ & $14.831 \pm 0.040$ & $14.009 \pm 0.040$ & $12.907 \pm 0.028$ & $14.738 \pm 0.025$ & $13.882 \pm 0.055$ & $12.901 \pm 0.026$ & \\
\hline 63 & PG $1512+370$ & $15.432 \pm 0.053$ & $14.702 \pm 0.064$ & $13.698 \pm 0.054$ & $15.477 \pm 0.132$ & $14.675 \pm 0.048$ & $13.569 \pm 0.070$ & \\
\hline 64 & PG $1534+580$ & $13.609 \pm 0.038$ & $12.906 \pm 0.041$ & $12.181 \pm 0.031$ & $13.041 \pm 0.038$ & $12.459 \pm 0.053$ & $11.754 \pm 0.049$ & ext \\
\hline 65 & PG $1543+489$ & $15.191 \pm 0.047$ & $14.287 \pm 0.046$ & $13.141 \pm 0.033$ & $15.122 \pm 0.032$ & $14.174 \pm 0.085$ & $13.114 \pm 0.036$ & \\
\hline 66 & PG $1545+210$ & $14.833 \pm 0.044$ & $14.180 \pm 0.054$ & $13.187 \pm 0.038$ & $14.465 \pm 0.106$ & $13.908 \pm 0.169$ & $12.918 \pm 0.098$ & ext \\
\hline 68 & B2 $1611+34$ & $16.239 \pm 0.086$ & $15.317 \pm 0.096$ & $14.717 \pm 0.079$ & $16.187 \pm 0.236$ & $15.612 \pm 0.199$ & $14.768 \pm 0.125$ & \\
\hline 69 & $3 \mathrm{C} 334$ & $15.552 \pm 0.062$ & $14.919 \pm 0.076$ & $14.088 \pm 0.050$ & $15.588 \pm 0.066$ & $14.754 \pm 0.093$ & $14.164 \pm 0.059$ & \\
\hline 70 & PG $1626+554$ & $14.406 \pm 0.042$ & $13.686 \pm 0.040$ & $12.675 \pm 0.031$ & $14.317 \pm 0.056$ & $13.600 \pm 0.100$ & $12.571 \pm 0.019$ & \\
\hline
\end{tabular}


Table 5

(Continued)

\begin{tabular}{|c|c|c|c|c|c|c|c|c|}
\hline \multirow[t]{2}{*}{ ID } & \multirow[t]{2}{*}{ Object } & \multicolumn{3}{|c|}{ Profile Fitting Magnitude } & \multicolumn{3}{|c|}{ Aperture Magnitude } & \multirow[t]{2}{*}{$\mathrm{Ext}^{\mathrm{a}}$} \\
\hline & & $J$ & $H$ & $K$ & $J$ & $H$ & $K$ & \\
\hline 71 & OS 562 & $14.909 \pm 0.041$ & $14.338 \pm 0.044$ & $13.540 \pm 0.040$ & $14.974 \pm 0.038$ & $14.350 \pm 0.072$ & $13.587 \pm 0.046$ & \\
\hline 73 & PG 1704+608 & $14.148 \pm 0.032$ & $13.473 \pm 0.038$ & $12.433 \pm 0.028$ & $14.083 \pm 0.031$ & $13.397 \pm 0.045$ & $12.391 \pm 0.026$ & \\
\hline 74 & MRK 506 & $13.277 \pm 0.040$ & $12.361 \pm 0.039$ & $11.650 \pm 0.031$ & $12.040 \pm 0.026$ & $11.292 \pm 0.034$ & $10.807 \pm 0.035$ & \\
\hline 75 & $4 C 34.47$ & $14.418 \pm 0.031$ & $13.821 \pm 0.032$ & $12.888 \pm 0.028$ & $14.370 \pm 0.041$ & $13.802 \pm 0.056$ & $12.869 \pm 0.054$ & \\
\hline 76 & $4 C 73.18$ & $14.287 \pm 0.035$ & $13.496 \pm 0.040$ & $12.488 \pm 0.031$ & $14.232 \pm 0.037$ & $13.416 \pm 0.032$ & $12.467 \pm 0.054$ & \\
\hline 79 & $4 C 31.63$ & $14.466 \pm 0.032$ & $13.458 \pm 0.028$ & $12.340 \pm 0.024$ & $14.369 \pm 0.004$ & $13.353 \pm 0.030$ & $12.267 \pm 0.008$ & \\
\hline 80 & PG 2214+139 & $13.277 \pm 0.042$ & $12.353 \pm 0.037$ & $11.341 \pm 0.024$ & $12.563 \pm 0.043$ & $11.758 \pm 0.038$ & $11.121 \pm 0.055$ & \\
\hline 81 & PKS 2216-038 & $14.617 \pm 0.036$ & $14.317 \pm 0.029$ & $13.838 \pm 0.053$ & $14.630 \pm 0.064$ & $14.279 \pm 0.078$ & $13.802 \pm 0.112$ & \\
\hline 82 & $3 \mathrm{C} 446$ & $15.549 \pm 0.039$ & $14.596 \pm 0.041$ & $13.609 \pm 0.039$ & $15.597 \pm 0.093$ & $14.843 \pm 0.070$ & $13.638 \pm 0.067$ & \\
\hline 83 & 4C 11.69 & $15.174 \pm 0.045$ & $14.556 \pm 0.063$ & $13.765 \pm 0.052$ & $15.198 \pm 0.077$ & $14.480 \pm 0.039$ & $13.760 \pm 0.133$ & \\
\hline 84 & PG $2251+113$ & $14.442 \pm 0.036$ & $13.473 \pm 0.036$ & $12.507 \pm 0.029$ & $14.339 \pm 0.024$ & $13.417 \pm 0.038$ & $12.451 \pm 0.036$ & \\
\hline
\end{tabular}

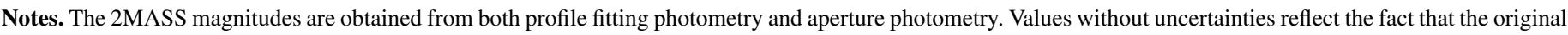
sources did not provide uncertainties.

a An "ext" indicates that the aperture magnitude is from the 2MASS extended source catalog, instead of point-source catalog.

0.5 (see Figure 1 in McLeod \& Rieke 1995) and is easier to detect.

For 33 sample members we have made our own observations, or used those from the literature, in order to determine the $H$-band host galaxy fractions. These are given in Table 6.

Our IRTF observations (5 objects), as well as those of McLeod \& Rieke (1994a, 1994b, 17 objects) used groundbased telescopes, long exposure times, and were obtained with seeing of 1-2 arcsec. In general, infrared imaging is done by mosaicking together large numbers of short exposure time images of the object on different positions on the chip. We reduced our data, generally 30 minute exposures on target, using the DIMSUM task inside IRAF. We determined our host-galaxy fractions using a similar one-dimensional analysis procedure to that of McLeod \& Rieke (1994a). This includes fitting a standard star observed just before or after the target to the one-dimensional surface brightness profile. Minimal and maximal subtraction of standard star point-spread functions (PSFs) indicates an uncertainty in this part of the procedure of just a few percent, which is small compared with other systematic uncertainties.

We observed an additional seven higher redshift sample members with NICMOS on $H S T$ with $H$-band, and supplement these with four more similar observations by McLeod \& McLeod (2001, two of these superseding results from McLeod $\&$ Rieke 1994b). For the sharper and more regular HST images, two-dimensional PSF fitting is possible. Our observations of the targets were for one orbit each and we also observed a standard star for each target. We used GALFIT (Peng et al. 2002) to fit the PSF to each image, along with several galaxy models (e.g., exponential disks and appropriately constrained Sérsic profiles), and took the results from the best fit. The different methods provided host galaxy fractions consistent to a few percent or better, again more than adequate for SED work.

We choose the 2MASS aperture magnitude (Table 5) as the total magnitude of an AGN and its host. It is straightforward to correct the $H$-band host galaxy contamination once we have the measured host fraction in the $H$ band. To correct $J$ - and $K$-band magnitudes for host galaxy contamination, we subtracted an appropriately scaled and redshifted elliptical galaxy template
(NGC 584 from Dale et al. 2007) from the 2MASS aperture photometry.

Table 7 gives the final AGN $J, H$, and $K$ magnitudes used for the SEDs. For the 33 objects with detailed host galaxy corrections, the corrected magnitudes are listed. For the rest of the objects, the 2MASS PSF magnitudes (profile fitting magnitudes) are used.

The red part of the optical spectra may also be affected by the host contamination, although not as much as in the NIR. We also tried to remove the host contribution when extracting the spectra as described in Section 3.5.

\subsection{Galactic Reddening Correction}

The FUV-to-optical spectra suffer from Galactic dust extinction. We corrected this with an empirical mean extinction law (Cardelli et al. 1989), assuming $R_{v}=A_{V} / E(B-V)=3.1$, a typical value for the diffuse interstellar medium. $E(B-V)$ is obtained from $\mathrm{NED}^{15}$ based on the dust map created by Schlegel et al. (1998).

The FUV-UV-optical spectra are combined first before applying the Galactic reddening correction. The FUV spectra from FUSE extend below $1000 \AA$, which the Cardelli et al. (1989) extinction curve does not cover. Shang et al. (2005) has shown a short extrapolation of the extinction curve below $1000 \AA$ is acceptable, and we use the same technique here.

\subsection{Measurements}

We have made detailed measurements of all the spectral properties (continua and emission lines), which need further analyses and will be presented in a separate paper. Here, we briefly describe the measurements and derived quantities related to this work. These quantities are listed in Table 1.

$$
\text { 4.3.1. Redshift }
$$

Since all our objects have high-quality UV-optical spectra, we used the optical narrow line [O III] $\lambda 5007$ to define the rest

\footnotetext{
15 NASA/IPAC Extragalactic Database (NED) is operated by the Jet

Propulsion Laboratory, California Institute of Technology, under contract with the National Aeronautics and Space Administration.
} 
Table 6

Total JHK Magnitudes and Observed Host Fraction

\begin{tabular}{|c|c|c|c|c|c|c|c|c|}
\hline \multirow{2}{*}{$\begin{array}{l}\text { ID } \\
\text { (1) }\end{array}$} & \multirow{2}{*}{$\begin{array}{c}\text { Object } \\
\text { (2) }\end{array}$} & \multicolumn{3}{|c|}{ Total Mag } & \multicolumn{3}{|c|}{ Host Fraction } & \multirow{2}{*}{$\begin{array}{c}\text { Reference }^{a} \\
\text { (9) }\end{array}$} \\
\hline & & $\begin{array}{c}J \\
(3)\end{array}$ & $\begin{array}{c}H \\
(4)\end{array}$ & $\begin{array}{c}K \\
(5)\end{array}$ & $\begin{array}{c}J \\
(6)\end{array}$ & $\begin{array}{c}H \\
(7)\end{array}$ & $\begin{array}{c}K \\
(8)\end{array}$ & \\
\hline 2 & PG $0052+251$ & 13.81 & 13.07 & 12.19 & 0.27 & 0.22 & 0.14 & $\mathrm{P} 2$ \\
\hline 13 & IRAS F07546+3928 & 12.89 & 11.97 & 11.00 & 0.73 & 0.53 & 0.29 & IRTF \\
\hline 15 & PG 0844+349 & 13.33 & 12.67 & 11.96 & 0.58 & 0.542 & 0.36 & $\mathrm{P} 1$ \\
\hline 20 & PG 0947+396 & 14.42 & 13.72 & 12.64 & 0.33 & 0.27 & 0.16 & P3 \\
\hline 21 & PG 0953+414 & 14.18 & 13.46 & 12.51 & 0.29 & 0.23 & 0.15 & $\mathrm{P} 2$ \\
\hline 24 & PG 1001+054 & 14.99 & 14.18 & 13.03 & 0.75 & 0.58 & 0.30 & $\operatorname{IRTF}(\mathrm{P} 1,<0.273)$ \\
\hline 28 & PG 1100+772 & 14.45 & 13.75 & 12.96 & 0.27 & 0.223 & 0.18 & HST \\
\hline 31 & PG 1114+445 & 14.15 & 13.43 & 12.29 & 0.54 & 0.461 & 0.23 & $\mathrm{P} 1$ \\
\hline 32 & PG $1115+407$ & 14.46 & 13.58 & 12.63 & 0.70 & 0.506 & 0.31 & $\mathrm{P} 1$ \\
\hline 33 & PG 1116+215 & 13.55 & 12.66 & 11.51 & 0.41 & 0.29 & 0.15 & $\mathrm{P} 2$ \\
\hline 39 & TEX $1156+213$ & 15.58 & 14.86 & 13.82 & 0.68 & 0.55 & 0.35 & IRTF \\
\hline 40 & PG $1202+281$ & 14.62 & 13.86 & 12.86 & 0.50 & 0.40 & 0.24 & $\mathrm{P} 2$ \\
\hline 42 & PG 1216+069 & 14.51 & 13.92 & 13.43 & 0.10 & 0.090 & 0.10 & HST \\
\hline 43 & PG $1226+023$ & 11.69 & 10.95 & 9.94 & 0.16 & 0.13 & 0.08 & $\mathrm{P} 2$ \\
\hline 46 & PG 1259+593 & 14.77 & 13.88 & 13.04 & 0.08 & 0.064 & 0.05 & HST \\
\hline 48 & PG 1309+355 & 14.09 & 13.50 & 12.59 & 0.35 & 0.33 & 0.22 & $\mathrm{P} 2$ \\
\hline 49 & PG $1322+659$ & 14.71 & 13.87 & 12.79 & 0.29 & 0.22 & 0.12 & P3 (P2, 0.43) \\
\hline 51 & PG $1351+640$ & 13.41 & 12.75 & 11.84 & 0.47 & 0.430 & 0.24 & $\mathrm{P} 1$ \\
\hline 53 & PG $1352+183$ & 14.88 & 14.18 & 13.09 & 0.55 & 0.48 & 0.26 & P3 \\
\hline 56 & PG $1402+261$ & 14.31 & 13.37 & 12.11 & 0.28 & 0.19 & 0.09 & $\mathrm{P} 2$ \\
\hline 57 & PG $1411+442$ & 13.25 & 12.46 & 11.47 & 0.50 & 0.408 & 0.22 & $\mathrm{P} 1$ \\
\hline 58 & PG $1415+451$ & 13.98 & 13.04 & 12.19 & 0.54 & 0.377 & 0.24 & $\mathrm{P} 1$ \\
\hline 59 & PG $1425+267$ & 15.11 & 14.30 & 13.54 & 0.28 & 0.214 & 0.18 & HST \\
\hline 60 & PG $1427+480$ & 15.10 & 14.62 & 13.43 & 0.26 & 0.26 & 0.14 & P3 (P2, 0.45) \\
\hline 62 & PG 1444+407 & 14.74 & 13.88 & 12.90 & 0.37 & 0.26 & 0.17 & $\mathrm{P} 2$ \\
\hline 63 & PG $1512+370$ & 15.48 & 14.68 & 13.57 & 0.29 & 0.224 & 0.13 & HST \\
\hline 64 & PG $1534+580$ & 13.04 & 12.46 & 11.75 & 0.46 & 0.46 & 0.29 & IRTF \\
\hline 65 & PG $1543+489$ & 15.12 & 14.17 & 13.11 & 0.15 & 0.105 & 0.06 & HST \\
\hline 66 & PG $1545+210$ & 14.47 & 13.91 & 12.92 & 0.56 & 0.52 & 0.34 & $\mathrm{P} 2$ \\
\hline 70 & PG 1626+554 & 14.32 & 13.60 & 12.57 & 0.53 & 0.456 & 0.25 & $\mathrm{P} 1$ \\
\hline 73 & PG 1704+608 & 14.08 & 13.40 & 12.39 & 0.25 & 0.217 & 0.14 & HST \\
\hline 80 & PG 2214+139 & 12.56 & 11.76 & 11.12 & 0.64 & 0.516 & 0.37 & $\mathrm{P} 1$ \\
\hline 85 & PG 2349-014 & 13.88 & 12.96 & 11.87 & 0.94 & 0.65 & 0.36 & IRTF \\
\hline
\end{tabular}

Notes. Total magnitude is adopted from 2MASS aperture magnitude in Table 5.

${ }^{a}$ Reference for adopted $H$-band host fraction. A reference ID and a number in a parenthesis indicate another $H$-band host fraction from the corresponding reference. P1: McLeod \& Rieke 1994a; P2: McLeod \& Rieke 1994b; P3: McLeod \& McLeod 2001; IRTF: our own observations from IRTF; HST: our own observations from HST NICMOS.

frame of each object, and double-checked against other strong narrow emission lines. The centroid of [O III] $\lambda 5007$ is obtained by fitting this spectral region with a power law for the local continuum and Gaussian components for different emission lines simultaneously (see Shang et al. 2005, 2007, for details). We can reach a redshift accuracy of 0.0002 for most objects.

When [O III] $\lambda 5007$ is weak or missing from our spectral coverage for some objects, we have obtained the redshift from NED as an initial guess in our spectral fitting, checked against the fitted centroids of other available strong emission lines, and made corrections when needed. The redshift uncertainty in this case is about 0.001, sufficient for SED work.

\subsubsection{Radio Loudness}

The traditional definition of radio loudness $R$ is the ratio of rest-frame flux density at radio $5 \mathrm{GHz}$ to that at optical $4400 \AA$, $R=f(5 \mathrm{GHz}) / f(4400 \AA)$, and $R=10$ separates $\mathrm{RL}$ and RQ objects. We use $f(4215 \AA)$, instead of $f(4400 \AA)$, because this local continuum is well defined (see Section 5.2) in our spectra and it makes little difference in calculating $R$. To obtain $f(5 \mathrm{GHz})$, we have interpolated for most objects using two radio measurements embracing $5 \mathrm{GHz}$ (rest frame) in frequency. For RQ objects, there is usually only one measurement around $5 \mathrm{GHz}$ in the observed frame, we therefore assume a flat spectral index (in $f_{v}$ ) and take the value as rest-frame $f(5 \mathrm{GHz})$ as well. Since all RQ objects have $z<0.5$, this will not cause a big error, especially when using $R$ to distinguish RL and RQ quasars.

\subsubsection{Luminosity}

The continuum luminosity is given as $\lambda L_{\lambda}(3000 \AA)$, measured at $3000 \AA$ rest-frame wavelength, and assuming a flat $\Lambda \mathrm{CDM}$ cosmology with $H_{0}=70 \mathrm{~km} \mathrm{~s}^{-1} \mathrm{Mpc}^{-1}, \Omega_{m}=0.3$, and $\Omega_{\Lambda}=0.7$. If desired, an average multiplicative correction factor of five, taken from Richards et al. (2006, Figure 12), can be applied to $\lambda L_{\lambda}(3000 \AA)$, to estimate the bolometric luminosity. Other more refined theoretical bolometric corrections can also be adopted from Nemmen \& Brotherton (2010).

We measure the bolometric luminosity for our individual quasars in various ways and report the results in a forthcoming paper (J. Runnoe et al. 2011, in preparation). On average, the bolometric luminosities are very similar to $5 \lambda L_{\lambda 3000}$. There are 
Table 7

Host Corrected $J H K$ Magnitude and Flux

\begin{tabular}{|c|c|c|c|c|c|c|c|c|}
\hline \multirow{2}{*}{$\begin{array}{l}\text { ID } \\
\text { (1) }\end{array}$} & \multirow{2}{*}{$\begin{array}{l}\text { Object } \\
\text { (2) }\end{array}$} & \multicolumn{3}{|c|}{ AGN Magnitude } & \multicolumn{3}{|c|}{ AGN Flux Density (mJy) } & \multirow{2}{*}{$\begin{array}{l}\text { Host } \\
\text { (9) }\end{array}$} \\
\hline & & $\begin{array}{c}J \\
(3)\end{array}$ & $\begin{array}{c}H \\
(4)\end{array}$ & $\begin{array}{c}K \\
(5)\end{array}$ & $\begin{array}{c}J \\
(6)\end{array}$ & $\begin{array}{c}H \\
(7)\end{array}$ & $\begin{array}{c}K \\
(8)\end{array}$ & \\
\hline 2 & PG $0052+251$ & 14.15 & 13.34 & 12.36 & $3.49 \pm 0.06$ & $4.72 \pm 0.09$ & $7.58 \pm 0.14$ & yes \\
\hline 3 & PKS 0112-017 & 16.93 & 15.81 & 15.66 & $0.27 \pm 0.04$ & $0.49 \pm 0.06$ & $0.36 \pm 0.07$ & \\
\hline 4 & $3 \mathrm{C} 37$ & 16.73 & 15.91 & 15.10 & $0.32 \pm 0.04$ & 0.44 & $0.61 \pm 0.08$ & \\
\hline 5 & $3 \mathrm{C} 47$ & 16.35 & 15.44 & 14.55 & $0.46 \pm 0.05$ & $0.68 \pm 0.07$ & $1.01 \pm 0.08$ & \\
\hline 6 & 4C 01.04 & 15.84 & 14.87 & 13.82 & $0.74 \pm 0.05$ & $1.15 \pm 0.09$ & $1.98 \pm 0.11$ & \\
\hline 7 & 4C 10.06 & 15.35 & 14.55 & 13.80 & $1.15 \pm 0.05$ & $1.55 \pm 0.07$ & $2.01 \pm 0.09$ & \\
\hline 8 & PKS 0403-13 & 15.61 & 14.82 & 14.12 & $0.91 \pm 0.05$ & $1.21 \pm 0.09$ & $1.50 \pm 0.08$ & \\
\hline 9 & $3 \mathrm{C} 110$ & 14.87 & 14.73 & 13.94 & $1.80 \pm 0.07$ & $1.31 \pm 0.06$ & $1.77 \pm 0.08$ & \\
\hline 10 & $3 \mathrm{C} 175$ & 14.88 & 14.68 & 14.00 & $1.78 \pm 0.11$ & $1.37 \pm 0.10$ & $1.67 \pm 0.09$ & \\
\hline 12 & B2 $0742+31$ & 14.48 & 13.78 & 12.95 & $2.57 \pm 0.07$ & $3.15 \pm 0.09$ & $4.40 \pm 0.12$ & \\
\hline 13 & IRAS F07546+3928 & 14.30 & 12.79 & 11.37 & $3.04 \pm 0.06$ & $7.84 \pm 0.14$ & $18.88 \pm 0.52$ & yes \\
\hline 14 & $3 \mathrm{C} 207$ & 16.69 & 15.96 & 15.04 & $0.34 \pm 0.05$ & $0.42 \pm 0.05$ & $0.64 \pm 0.07$ & \\
\hline 15 & PG 0844+349 & 14.28 & 13.52 & 12.44 & $3.09 \pm 0.03$ & $4.00 \pm 0.04$ & $7.05 \pm 0.06$ & yes \\
\hline 16 & PKS 0859-14 & 15.73 & 14.85 & 14.78 & $0.81 \pm 0.06$ & $1.18 \pm 0.06$ & $0.82 \pm 0.09$ & \\
\hline 17 & $3 \mathrm{C} 215$ & 16.86 & 15.85 & 15.05 & $0.29 \pm 0.04$ & $0.47 \pm 0.07$ & $0.64 \pm 0.06$ & \\
\hline 18 & 4C 39.25 & 15.34 & 14.85 & 14.00 & $1.17 \pm 0.05$ & $1.18 \pm 0.08$ & $1.67 \pm 0.08$ & \\
\hline 19 & $4 \mathrm{C} 40.24$ & 16.71 & 16.19 & 15.38 & $0.33 \pm 0.05$ & $0.34 \pm 0.08$ & $0.47 \pm 0.09$ & \\
\hline 20 & PG 0947+396 & 14.84 & 14.06 & 12.82 & $1.85 \pm 0.12$ & $2.43 \pm 0.18$ & $4.97 \pm 0.27$ & yes \\
\hline 21 & PG 0953+414 & 14.55 & 13.74 & 12.69 & $2.41 \pm 0.07$ & $3.27 \pm 0.15$ & $5.60 \pm 0.21$ & yes \\
\hline 22 & 4C 55.17 & 15.68 & 14.94 & 14.19 & $0.85 \pm 0.05$ & $1.08 \pm 0.08$ & $1.41 \pm 0.08$ & \\
\hline 23 & $3 \mathrm{C} 232$ & 14.94 & 14.44 & 13.76 & $1.68 \pm 0.06$ & $1.72 \pm 0.08$ & $2.09 \pm 0.08$ & \\
\hline 24 & PG 1001+054 & 16.50 & 15.12 & 13.42 & $0.40 \pm 0.02$ & $0.92 \pm 0.06$ & $2.86 \pm 0.11$ & yes \\
\hline 25 & $4 C 22.26$ & 16.78 & 16.58 & 15.65 & $0.31 \pm 0.05$ & 0.24 & $0.37 \pm 0.06$ & \\
\hline 26 & $4 \mathrm{C} 41.21$ & 15.36 & 14.70 & 13.96 & $1.14 \pm 0.06$ & $1.35 \pm 0.09$ & $1.74 \pm 0.10$ & \\
\hline 27 & 4C 20.24 & 16.03 & 15.24 & 14.61 & $0.62 \pm 0.04$ & $0.82 \pm 0.06$ & $0.95 \pm 0.06$ & \\
\hline 28 & PG $1100+772$ & 14.79 & 14.03 & 13.17 & $1.93 \pm 0.07$ & $2.50 \pm 0.12$ & $3.60 \pm 0.10$ & yes \\
\hline 29 & PG 1103-006 & 15.31 & 14.79 & 13.85 & $1.20 \pm 0.06$ & $1.24 \pm 0.07$ & $1.92 \pm 0.11$ & \\
\hline 30 & $3 \mathrm{C} 254$ & 15.88 & 15.29 & 14.67 & $0.71 \pm 0.05$ & $0.78 \pm 0.07$ & $0.90 \pm 0.09$ & \\
\hline 31 & PG 1114+445 & 15.00 & 14.10 & 12.58 & $1.59 \pm 0.03$ & $2.35 \pm 0.13$ & $6.19 \pm 0.11$ & yes \\
\hline 32 & PG 1115+407 & 15.75 & 14.35 & 13.03 & $0.80 \pm 0.02$ & $1.86 \pm 0.05$ & $4.09 \pm 0.11$ & yes \\
\hline 33 & PG 1116+215 & 14.12 & 13.03 & 11.69 & $3.58 \pm 0.03$ & $6.28 \pm 0.12$ & $14.06 \pm 0.13$ & yes \\
\hline 35 & PKS 1127-14 & 15.28 & 14.43 & 13.59 & $1.23 \pm 0.07$ & $1.73 \pm 0.08$ & $2.44 \pm 0.11$ & \\
\hline 36 & $3 \mathrm{C} 263$ & 14.83 & 14.39 & 13.65 & $1.86 \pm 0.07$ & $1.80 \pm 0.07$ & $2.31 \pm 0.09$ & \\
\hline 37 & MC2 1146+111 & 16.57 & 16.07 & 15.60 & $0.38 \pm 0.05$ & $0.38 \pm 0.07$ & $0.38 \pm 0.09$ & \\
\hline 38 & 4C 49.22 & 15.80 & 14.95 & 13.81 & $0.76 \pm 0.04$ & $1.07 \pm 0.08$ & $1.99 \pm 0.07$ & \\
\hline 39 & TEX 1156+213 & 16.80 & 15.73 & 14.29 & $0.30 \pm 0.02$ & $0.52 \pm 0.03$ & $1.28 \pm 0.13$ & yes \\
\hline 40 & PG 1202+281 & 15.37 & 14.41 & 13.15 & $1.13 \pm 0.04$ & $1.76 \pm 0.06$ & $3.66 \pm 0.10$ & yes \\
\hline 41 & $4 \mathrm{C} 64.15$ & 16.74 & 15.80 & 15.46 & $0.32 \pm 0.05$ & $0.49 \pm 0.08$ & $0.44 \pm 0.07$ & \\
\hline 42 & PG 1216+069 & 14.63 & 14.02 & 13.54 & $2.24 \pm 0.12$ & $2.53 \pm 0.23$ & $2.56 \pm 0.09$ & yes \\
\hline 43 & PG 1226+023 & 11.88 & 11.10 & 10.02 & $28.22 \pm 0.52$ & $37.18 \pm 0.68$ & $65.45 \pm 1.21$ & yes \\
\hline 45 & 3C 277.1 & 16.48 & 16.15 & 14.98 & $0.41 \pm 0.04$ & $0.36 \pm 0.06$ & $0.68 \pm 0.08$ & \\
\hline 46 & PG 1259+593 & 14.86 & 13.95 & 13.10 & $1.81 \pm 0.12$ & $2.69 \pm 0.12$ & $3.84 \pm 0.07$ & yes \\
\hline 47 & $3 \mathrm{C} 281$ & 16.37 & 15.92 & 15.09 & $0.45 \pm 0.06$ & $0.44 \pm 0.08$ & $0.61 \pm 0.11$ & \\
\hline 48 & PG 1309+355 & 14.56 & 13.94 & 12.85 & $2.39 \pm 0.02$ & $2.72 \pm 0.03$ & $4.83 \pm 0.09$ & yes \\
\hline 49 & PG 1322+659 & 15.08 & 14.14 & 12.93 & $1.48 \pm 0.05$ & $2.26 \pm 0.21$ & $4.49 \pm 0.12$ & yes \\
\hline 50 & 3C 288.1 & 16.31 & 16.35 & 15.52 & $0.48 \pm 0.05$ & $0.30 \pm 0.06$ & $0.41 \pm 0.06$ & \\
\hline 51 & PG $1351+640$ & 14.09 & 13.36 & 12.14 & $3.69 \pm 0.03$ & $4.64 \pm 0.13$ & $9.29 \pm 0.09$ & yes \\
\hline 53 & PG $1352+183$ & 15.76 & 14.89 & 13.41 & $0.79 \pm 0.06$ & $1.13 \pm 0.02$ & $2.88 \pm 0.11$ & yes \\
\hline 54 & 4C 19.44 & 14.86 & 14.69 & 13.89 & $1.81 \pm 0.07$ & $1.36 \pm 0.10$ & $1.85 \pm 0.09$ & \\
\hline 55 & 4C 58.29 & 15.95 & 15.05 & 15.06 & $0.66 \pm 0.05$ & $0.98 \pm 0.07$ & $0.63 \pm 0.08$ & \\
\hline 56 & PG $1402+261$ & 14.66 & 13.60 & 12.21 & $2.18 \pm 0.06$ & $3.72 \pm 0.14$ & $8.71 \pm 0.16$ & yes \\
\hline 57 & PG $1411+442$ & 14.00 & 13.03 & 11.74 & $4.00 \pm 0.04$ & $6.28 \pm 0.06$ & $13.43 \pm 0.12$ & yes \\
\hline 58 & PG $1415+451$ & 14.81 & 13.55 & 12.49 & $1.90 \pm 0.05$ & $3.89 \pm 0.11$ & $6.73 \pm 0.19$ & yes \\
\hline 59 & PG 1425+267 & 15.47 & 14.57 & 13.75 & $1.03 \pm 0.08$ & $1.52 \pm 0.13$ & $2.11 \pm 0.10$ & yes \\
\hline 60 & PG $1427+480$ & 15.42 & 14.95 & 13.60 & $1.08 \pm 0.08$ & $1.07 \pm 0.09$ & $2.42 \pm 0.16$ & yes \\
\hline 61 & PG $1440+356$ & 12.94 & 12.00 & 11.06 & $10.63 \pm 0.29$ & $16.23 \pm 0.30$ & $25.11 \pm 0.46$ & \\
\hline 62 & PG 1444+407 & 15.24 & 14.21 & 13.11 & $1.28 \pm 0.04$ & $2.12 \pm 0.10$ & $3.80 \pm 0.11$ & yes \\
\hline 63 & PG $1512+370$ & 15.85 & 14.95 & 13.72 & $0.73 \pm 0.09$ & $1.07 \pm 0.05$ & $2.17 \pm 0.14$ & yes \\
\hline 64 & PG 1534+580 & 13.71 & 13.13 & 12.12 & $5.23 \pm 0.19$ & $5.73 \pm 0.26$ & $9.46 \pm 0.44$ & yes \\
\hline 65 & PG $1543+489$ & 15.30 & 14.29 & 13.19 & $1.21 \pm 0.03$ & $1.97 \pm 0.16$ & $3.53 \pm 0.13$ & yes \\
\hline 66 & PG $1545+210$ & 15.36 & 14.70 & 13.37 & $1.14 \pm 0.12$ & $1.35 \pm 0.21$ & $2.99 \pm 0.28$ & yes \\
\hline 68 & B2 1611+34 & 16.24 & 15.32 & 14.72 & $0.51 \pm 0.04$ & $0.76 \pm 0.07$ & $0.86 \pm 0.06$ & \\
\hline 69 & 3C 334 & 15.55 & 14.92 & 14.09 & $0.96 \pm 0.05$ & $1.10 \pm 0.08$ & $1.54 \pm 0.07$ & \\
\hline
\end{tabular}


Table 7

(Continued)

\begin{tabular}{|c|c|c|c|c|c|c|c|c|}
\hline \multirow{2}{*}{$\begin{array}{l}\text { ID } \\
\text { (1) } \\
\end{array}$} & \multirow{2}{*}{$\begin{array}{c}\text { Object } \\
\text { (2) }\end{array}$} & \multicolumn{3}{|c|}{ AGN Magnitude } & \multicolumn{3}{|c|}{ AGN Flux Density (mJy) } & \multirow{2}{*}{$\begin{array}{r}\text { Host } \\
\text { (9) }\end{array}$} \\
\hline & & $\begin{array}{c}J \\
(3) \\
\end{array}$ & $\begin{array}{c}H \\
(4)\end{array}$ & $\begin{array}{c}K \\
(5)\end{array}$ & $\begin{array}{c}J \\
(6)\end{array}$ & $\begin{array}{c}H \\
(7)\end{array}$ & $\begin{array}{c}K \\
(8)\end{array}$ & \\
\hline 70 & PG $1626+554$ & 15.14 & 14.26 & 12.89 & $1.40 \pm 0.08$ & $2.02 \pm 0.19$ & $4.66 \pm 0.09$ & yes \\
\hline 71 & OS 562 & 14.91 & 14.34 & 13.54 & $1.73 \pm 0.06$ & $1.88 \pm 0.07$ & $2.56 \pm 0.09$ & \\
\hline 72 & PKS 1656+053 & 15.32 & 14.98 & 14.17 & $1.19 \pm 0.07$ & $1.04 \pm 0.10$ & $1.43 \pm 0.11$ & \\
\hline 73 & PG 1704+608 & 14.40 & 13.66 & 12.56 & $2.77 \pm 0.08$ & $3.52 \pm 0.16$ & $6.31 \pm 0.17$ & yes \\
\hline 74 & MRK 506 & 13.28 & 12.36 & 11.65 & $7.77 \pm 0.29$ & $11.65 \pm 0.43$ & $14.59 \pm 0.40$ & \\
\hline 75 & $4 C 34.47$ & 14.42 & 13.82 & 12.89 & $2.72 \pm 0.08$ & $3.04 \pm 0.08$ & $4.66 \pm 0.13$ & \\
\hline 76 & $4 C 73.18$ & 14.29 & 13.50 & 12.49 & $3.07 \pm 0.11$ & $4.08 \pm 0.15$ & $6.73 \pm 0.19$ & \\
\hline 77 & MRK 509 & 12.00 & 11.12 & 10.19 & $25.26 \pm 0.93$ & $36.50 \pm 1.01$ & $55.97 \pm 1.55$ & \\
\hline 78 & 4C 06.69 & 14.60 & 14.15 & 13.38 & $2.30 \pm 0.06$ & $2.24 \pm 0.08$ & $2.96 \pm 0.11$ & \\
\hline 79 & $4 C 31.63$ & 14.47 & 13.46 & 12.34 & $2.60 \pm 0.07$ & $4.23 \pm 0.12$ & $7.73 \pm 0.14$ & \\
\hline 80 & PG 2214+139 & 13.66 & 12.55 & 11.61 & $5.48 \pm 0.20$ & $9.78 \pm 0.36$ & $15.13 \pm 0.70$ & yes \\
\hline 81 & PKS 2216-038 & 14.62 & 14.32 & 13.84 & $2.26 \pm 0.08$ & $1.92 \pm 0.05$ & $1.94 \pm 0.09$ & \\
\hline 82 & $3 \mathrm{C} 446$ & 15.55 & 14.60 & 13.61 & $0.96 \pm 0.04$ & $1.48 \pm 0.05$ & $2.40 \pm 0.09$ & \\
\hline 83 & 4C 11.69 & 15.17 & 14.56 & 13.77 & $1.36 \pm 0.06$ & $1.54 \pm 0.08$ & $2.07 \pm 0.10$ & \\
\hline 84 & PG $2251+113$ & 14.44 & 13.47 & 12.51 & $2.67 \pm 0.10$ & $4.19 \pm 0.15$ & $6.61 \pm 0.18$ & \\
\hline 85 & PG 2349-014 & 16.98 & 14.10 & 12.36 & $0.26 \pm 0.02$ & $2.35 \pm 0.19$ & $7.58 \pm 0.42$ & yes \\
\hline
\end{tabular}

Notes. For objects without $H$-band host fraction, we take 2MASS profile fitting magnitude as the AGN magnitude. Values without uncertainties reflect the fact that the original sources did not provide uncertainties.

a Indicating whether we have host fraction from $H$-band observation.

a number of issues to consider in making bolometric corrections and caution is advised.

\section{SEDs}

\subsection{SEDs for Individual Objects}

With multiwavelength data in hand, it is very straightforward to combine the data to build the SEDs for individual objects (Figure 4). FUV-to-optical spectra are rebinned in the observed frame to a lower resolution, but not so much that the emissionline features are degraded too much. The bin size is $10 \AA$, corresponding to $1000 \mathrm{~km} \mathrm{~s}^{-1}$ at $1000 \AA$, and $500 \mathrm{~km} \mathrm{~s}^{-1}$ at 6000 A. Our Spitzer IRS mid-IR spectra have low resolution and sampling of $\gtrsim 0.02 \mu \mathrm{m}$, so we retain this sampling in the SEDs without invoking further rebinning. The rebuilt X-ray spectra have a sampling of $0.1 \mathrm{keV}$ (Section 3.7).

When we combined FUV-UV-optical spectra, we scaled data to photometric nights or HST observations (Shang et al. 2005, 2007). When photometric spectra overlapped, the agreement was better than 5\% (e.g., between ground-based and HST spectra, as well as inter-compared optical spectra).

We present the data in $f_{v}$ versus frequency (Hertz) and convert the flux density in each waveband to the same units of mJy $\left(10^{-26} \mathrm{erg} \mathrm{s}^{-1} \mathrm{~cm}^{-2} \mathrm{~Hz}^{-1}\right)$. After combining all the data, we apply a redshift correction to obtain the rest-frame SEDs. Only wavelength and frequency are shifted to the rest frame and the flux densities are left unchanged from the observed frame.

As shown in Figure 4, while we try to collect a uniform data set as much as possible, not all objects have data in all wavelength bands except for the UV-to-optical. Tables 3-9 give further information.

\subsection{Composite SEDs of $R L$ and $R Q$ Objects}

One of the main motivations of this study is to update the mean quasar SEDs of Elvis et al. (1994) using data from modern telescopes of higher sensitivity and better resolution.

We divided the sample into RL and RQ samples. For each sample, we first normalized the flux density of each object at rest-frame $4215 \AA$ where, after visual inspection of all spectra, there seems to be no strong emission features. The actual normalization factor is the mean flux density within $30 \AA$ around $4215 \AA$. The bandpass is chosen to be small, to avoid emission features, and large enough to minimize the noise in calculating the mean. For seven higher redshift RL objects, their rest-frame spectra do not cover $4215 \AA$. We therefore normalize them at $2200 \AA$, another continuum region, to a composite spectrum built with all spectra normalized earlier at $4215 \AA$ in the same sample. The normalization factor is derived from the mean flux density within $50 \AA$ around $2200 \AA$ in this case.

After normalization, we visually check the distribution of all the points from all objects and define the final bins in each waveband for calculating the composite SEDs. Each bin contributes one point in the final composite SED and the central frequency of each bin represents the final frequency of that point in the SEDs.

For each waveband with photometric points (radio, FIR, NIR), we locate a logarithmic frequency range (rest frame) to enclose all points and then define a few bins with equal bin size within the range. Since radio data span a large frequency range, sometimes there are obvious gaps in the distribution. In such cases, we define more than one frequency range to avoid the gaps and still try to keep similar bin size across the ranges. Figure 5 shows an example of defining bins for the RL sample.

For spectroscopic data (UV-optical, MIR, X-ray), it is easy to define consecutive bins with the same bin size. The bin size is chosen to have enough points in each bin for statistical significance and still be able to preserve the emission features. Table 10 lists the parameters we use to define the bins for each waveband.

After having defined the bins for a sample of interest, we rebin the data to obtain one single value for each bin. This is mostly necessary for spectroscopic data, and this is done for each object separately so that all objects with available data will have equal weights in building the composite SED. Two RQ objects have upper limits in the highest radio frequency bin and six objects each for RL and RQ samples have upper 

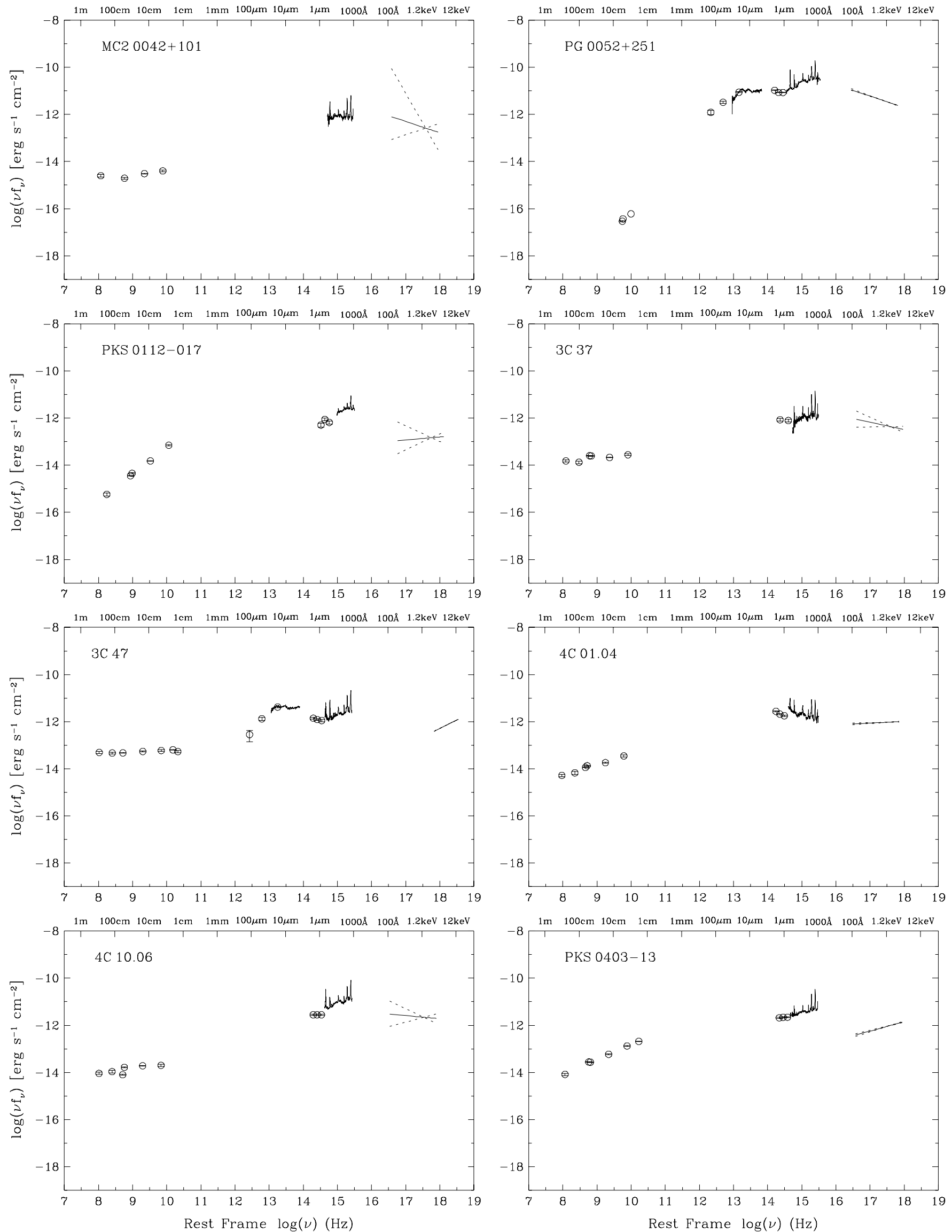

Figure 4. SEDs of the quasar sample, ordered by R.A. to match Table 1. The flux $\left(v f_{v}\right)$ is in the observed frame. Figures 4.1-4.11 are available in the online version of the journal.

(An extended version of this figure is available in the online journal.) 


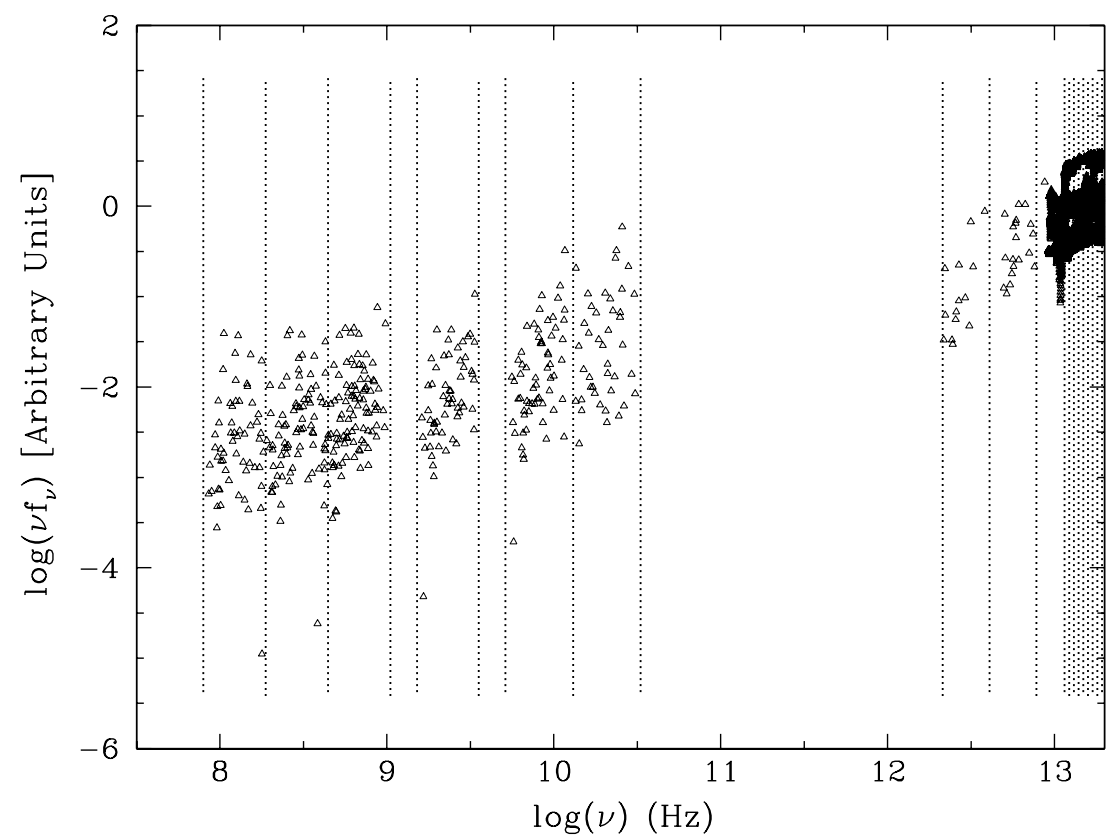

Figure 5. Example of defining bins in constructing composite SEDs for the RL sample. Open triangles are all data points from all RL objects. Vertical dotted lines define the bins, each of which contributes one point in the final composite SED. Note the two small gaps with no data point between $\log (v)$ of 9 and 10 . Shown on the right are the two FIR bins and MIR spectra where the bin size is much smaller.

Table 8

Far-UV FUSE Data

\begin{tabular}{|c|c|c|c|c|}
\hline ID & Object & Rest & $\mathrm{h}(\AA)^{\mathrm{a}}$ & Flux $^{b}$ \\
\hline (1) & (2) & $\begin{array}{l}\lambda 1 \\
(3)\end{array}$ & $\begin{array}{l}\lambda 2 \\
(4)\end{array}$ & $\begin{array}{c}\left(10^{-14} \mathrm{erg} \mathrm{s}^{-1} \mathrm{~cm}^{-2} \AA^{-1}\right) \\
(5)\end{array}$ \\
\hline 2 & PG $0052+251$ & 789 & 1028 & 1.67 \\
\hline 13 & IRAS F07546+3928 & 832 & 1083 & 0.72 \\
\hline 15 & PG 0844+349 & 856 & 1115 & 4.83 \\
\hline 20 & PG $0947+396$ & 758 & 984 & 1.12 \\
\hline 21 & PG $0953+414$ & 740 & 962 & 5.84 \\
\hline 28 & PG $1100+772$ & 696 & 905 & 1.50 \\
\hline 33 & PG $1116+215$ & 768 & 1010 & 6.58 \\
\hline 36 & $3 \mathrm{C} 263$ & 549 & 721 & 1.19 \\
\hline 42 & PG $1216+069$ & 678 & 892 & 1.16 \\
\hline 43 & PG $1226+023$ & 787 & 1025 & 27.36 \\
\hline 46 & PG $1259+593$ & 618 & 803 & 1.90 \\
\hline 49 & PG $1322+659$ & 781 & 1015 & 1.21 \\
\hline 51 & PG $1351+640$ & 837 & 1090 & 1.91 \\
\hline 56 & PG $1402+261$ & 776 & 1020 & 3.15 \\
\hline 58 & PG $1415+451$ & 811 & 1066 & 1.27 \\
\hline 61 & PG $1440+356$ & 839 & 1103 & 5.27 \\
\hline 64 & PG $1534+580$ & 885 & 1151 & 3.29 \\
\hline 70 & PG $1626+554$ & 798 & 1050 & 0.67 \\
\hline 73 & PG 1704+608 & 664 & 864 & 0.16 \\
\hline 74 & MRK 506 & 874 & 1138 & 1.74 \\
\hline 75 & 4C 34.47 & 757 & 984 & 0.60 \\
\hline 77 & MRK 509 & 885 & 1145 & 11.01 \\
\hline 80 & PG 2214+139 & 855 & 1113 & 2.23 \\
\hline 85 & PG 2349-014 & 777 & 1011 & 1.84 \\
\hline
\end{tabular}

Notes.

${ }^{\text {a }}$ Wavelength coverage in the rest frame.

b Mean flux density between 1050 and $1070 \AA$ in the observed frame with an uncertainty less than $5 \%$. There is not a common region in the rest frame where all objects have fluxes from FUSE.

limits in the MIPS $160 \mu \mathrm{m}$ band. They are included in the median combining process but none of the upper limits has a flux higher than the median value in the corresponding bin, therefore their uncertainty does not affect the composite SED.
This median combining is very effective in rejecting outliers and preventing any extreme objects from dominating the final SEDs. We therefore also refer to our composite SEDs as median SEDs. Finally, if one bin has less than eight points (i.e., objects), 
Table 9

X-Ray Spectral Parameters (Single or Broken Power Law)

\begin{tabular}{|c|c|c|c|c|c|c|c|c|c|}
\hline \multirow{2}{*}{$\begin{array}{l}\text { ID } \\
\text { (1) }\end{array}$} & \multirow{2}{*}{$\begin{array}{l}\text { Object } \\
\text { (2) }\end{array}$} & \multirow{2}{*}{$\begin{array}{l}\text { E1 } \\
\text { (3) }\end{array}$} & \multirow{2}{*}{$\begin{array}{l}\text { E2 } \\
\text { (4) }\end{array}$} & \multirow{2}{*}{$\begin{array}{l}\text { E3 } \\
\text { (5) }\end{array}$} & \multicolumn{4}{|c|}{$f_{v}=f_{0} E^{\alpha}$} & \multirow{2}{*}{$\begin{array}{c}\text { Reference } \\
\text { (10) }\end{array}$} \\
\hline & & & & & $\begin{array}{l}f_{0} \\
(6)\end{array}$ & $\begin{array}{c}\alpha \\
(7)\end{array}$ & $\begin{array}{l}f_{0} \\
(8)\end{array}$ & $\begin{array}{c}\alpha \\
(9)\end{array}$ & \\
\hline 1 & MC2 0042+101 & 0.1 & & 2.4 & $1.09 \mathrm{E}-4$ & $-1.48_{-2.04}^{+0.97}$ & & & $\mathrm{R}, \mathrm{Br} 97$ \\
\hline 2 & PG $0052+251$ & 0.1 & & 2.4 & $1.50 \mathrm{E}-3$ & $-1.49_{-0.04}^{+0.02}$ & & & R,Yu98 \\
\hline 3 & PKS 0112-017 & 0.1 & & 2.4 & $6.06 \mathrm{E}-5$ & $-0.88_{-0.79}^{+0.56}$ & & & $\mathrm{R}, \mathrm{Br} 97$ \\
\hline 4 & $3 C 37$ & 0.1 & & 2.4 & $1.78 \mathrm{E}-4$ & $-1.32_{-0.34}^{+0.34}$ & & & $\mathrm{R}, \mathrm{Br} 97$ \\
\hline 5 & $3 \mathrm{C} 47$ & 2.0 & & 10.0 & $9.93 \mathrm{E}-5$ & -0.28 & & & $\mathrm{C}, \mathrm{Sh} 05$ \\
\hline 6 & 4C 01.04 & 0.1 & & 2.4 & $3.90 \mathrm{E}-4$ & $-0.93_{-0.04}^{+0.04}$ & & & $\mathrm{R}, \mathrm{Br} 97$ \\
\hline 7 & 4C 10.06 & 0.1 & & 2.4 & $9.08 \mathrm{E}-4$ & $-1.13_{-0.56}^{+0.52}$ & & & $\mathrm{R}, \mathrm{Br} 97$ \\
\hline 8 & PKS 0403-13 & 0.1 & & 2.4 & $4.03 \mathrm{E}-4$ & $-0.60_{-0.06}^{+0.06}$ & & & $\mathrm{R}, \mathrm{Br} 97$ \\
\hline 9 & $3 \mathrm{C} 110$ & 0.1 & & 2.4 & $3.12 \mathrm{E}-4$ & $-1.48_{-0.08}^{+0.06}$ & & & $\mathrm{R}, \mathrm{Br} 97$ \\
\hline 11 & 3C 186 & 0.5 & & 8.0 & $4.59 \mathrm{E}-5$ & $-1.09_{-0.08}^{+0.08}$ & & & $\mathrm{C}, \mathrm{Si} 08$ \\
\hline 12 & B2 $0742+31$ & 0.1 & & 2.4 & $4.54 \mathrm{E}-4$ & $-0.56_{-0.68}^{+0.52}$ & & & $\mathrm{R}, \mathrm{Br} 97$ \\
\hline 13 & IRAS F07546+3928 & 0.1 & & 2.4 & $1.46 \mathrm{E}-4$ & $-2.16_{-0.34}^{+0.33}$ & & & $\mathrm{R}, \mathrm{Yu} 98$ \\
\hline 14 & $3 \mathrm{C} 207$ & 0.5 & & 8.0 & $2.39 \mathrm{E}-5$ & $-0.27_{-0.05}^{+0.05}$ & & & $\mathrm{C}, \mathrm{Be} 06$ \\
\hline 15 & PG 0844+349 & 0.3 & & 10.0 & $2.19 \mathrm{E}-3$ & $-1.24_{-0.03}^{+0.03}$ & & & $\mathrm{X}, \mathrm{Pi} 05$ \\
\hline 16 & PKS 0859-14 & 0.1 & & 2.4 & $5.05 \mathrm{E}-5$ & $-2.15_{-1.24}^{+0.90}$ & & & $\mathrm{R}, \mathrm{Br} 97$ \\
\hline 17 & $3 C 215$ & 0.3 & & 8.0 & $2.31 \mathrm{E}-4$ & $-0.80_{-0.11}^{+0.11}$ & & & $\mathrm{C}, \mathrm{HaO6}$ \\
\hline 18 & 4C 39.25 & 0.1 & & 2.4 & $5.83 \mathrm{E}-4$ & $-1.25_{-0.06}^{+0.06}$ & & & $\mathrm{R}, \mathrm{Br} 97$ \\
\hline 19 & 4C 40.24 & 0.1 & & 2.4 & $6.86 \mathrm{E}-5$ & $-0.82_{-0.86}^{+0.76}$ & & & $\mathrm{R}, \mathrm{Br} 97$ \\
\hline 20 & PG 0947+396 & 0.3 & 2.28 & 10.0 & $5.39 \mathrm{E}-4$ & $-1.41_{-0.03}^{+0.03}$ & $3.13 \mathrm{E}-4$ & $-0.75_{-0.09}^{+0.09}$ & X,Po04 \\
\hline 21 & PG 0953+414 & 0.3 & 1.77 & 10.0 & $1.11 \mathrm{E}-3$ & $-1.60_{-0.02}^{+0.05}$ & $7.99 \mathrm{E}-4$ & $-1.02_{-0.07}^{+0.07}$ & X,Po04 \\
\hline 22 & 4C 55.17 & 0.3 & & 10.0 & $8.66 \mathrm{E}-5$ & $-0.92_{-0.02}^{+0.04}$ & & & $\mathrm{C}, \mathrm{Ta} 07$ \\
\hline 23 & $3 C 232$ & 0.1 & & 2.4 & $4.24 \mathrm{E}-5$ & $-0.46_{-0.25}^{+0.20}$ & & & $\mathrm{R}, \mathrm{Br} 97$ \\
\hline 24 & PG $1001+054$ & 0.2 & & 2.0 & $3.18 \mathrm{E}-6$ & $-2.80_{-0.58}^{+1.07}$ & & & R,La97 \\
\hline 25 & 4C 22.26 & 0.1 & & 2.4 & $6.78 \mathrm{E}-5$ & $-1.71_{-0.81}^{+0.89}$ & & & $\mathrm{R}, \mathrm{Br} 97$ \\
\hline 26 & 4C 41.21 & 0.1 & & 2.4 & $4.25 \mathrm{E}-4$ & $-1.00_{-0.15}^{+0.15}$ & & & $\mathrm{R}, \mathrm{Br} 97$ \\
\hline 27 & 4C 20.24 & 0.5 & & 7.0 & $1.75 \mathrm{E}-4$ & $-0.58_{-0.01}^{+0.01}$ & & & $\mathrm{C}, \mathrm{CSC}$ \\
\hline 28 & PG $1100+772$ & 0.3 & & 10.0 & $1.21 \mathrm{E}-3$ & $-0.84_{-0.02}^{+0.03}$ & & & X,Pi05 \\
\hline 30 & $3 \mathrm{C} 254$ & 0.5 & & 8.0 & $9.28 \mathrm{E}-6$ & $-0.64_{-0.10}^{+0.11}$ & & & $\mathrm{C}, \mathrm{Be} 06$ \\
\hline 31 & PG 1114+445 & 0.3 & 2.00 & 10.0 & $2.56 \mathrm{E}-4$ & $-0.56_{-0.04}^{+0.09}$ & $2.51 \mathrm{E}-4$ & $-0.53_{-0.03}^{+0.03}$ & X,Po04 \\
\hline 32 & PG 1115+407 & 0.3 & 2.04 & 10.0 & $6.48 \mathrm{E}-4$ & $-1.85_{-0.02}^{+0.06}$ & $4.05 \mathrm{E}-4$ & $-1.19_{-0.10}^{+0.10}$ & X,Po04 \\
\hline 33 & PG $1116+215$ & 0.3 & 1.76 & 10.0 & $1.47 \mathrm{E}-3$ & $-1.72_{-0.03}^{+0.18}$ & $1.08 \mathrm{E}-3$ & $-1.17_{-0.11}^{+0.11}$ & X,Po04 \\
\hline 34 & 4C 12.40 & 0.1 & & 2.4 & $2.66 \mathrm{E}-5$ & $-1.98_{-1.04}^{+1.52}$ & & & $\mathrm{R}, \mathrm{Br} 97$ \\
\hline 35 & PKS 1127-14 & 0.5 & & 8.0 & $3.49 \mathrm{E}-4$ & $-0.20_{-0.03}^{+0.03}$ & & & $\mathrm{C}, \mathrm{Si} 08$ \\
\hline 36 & $3 C 263$ & 0.5 & & 8.0 & $7.29 \mathrm{E}-6$ & $-0.88_{-0.10}^{+0.10}$ & & & $\mathrm{C}, \mathrm{Be} 06$ \\
\hline 38 & 4C 49.22 & 0.5 & 1.85 & 8.0 & $5.71 \mathrm{E}-4$ & $-1.10_{-0.08}^{+0.08}$ & $4.52 \mathrm{E}-4$ & $-0.72_{-0.05}^{+0.05}$ & $\mathrm{C}, \mathrm{Sa} 06$ \\
\hline 40 & PG $1202+281$ & 0.3 & 1.76 & 10.0 & $8.32 \mathrm{E}-4$ & $-1.29_{-0.04}^{+0.04}$ & $5.99 \mathrm{E}-4$ & $-0.71_{-0.06}^{+0.06}$ & X,Po04 \\
\hline 42 & PG 1216+069 & 0.3 & 1.35 & 10.0 & $3.91 \mathrm{E}-4$ & $-1.90_{-0.30}^{+0.30}$ & $2.65 \mathrm{E}-4$ & $-0.60_{-0.30}^{+0.10}$ & $\mathrm{X}, \mathrm{Pi} 05$ \\
\hline 43 & PG $1226+023$ & 0.4 & 1.48 & 10.0 & $1.37 \mathrm{E}-2$ & $-0.94_{-0.01}^{+0.01}$ & $1.25 \mathrm{E}-2$ & $-0.70_{-0.01}^{+0.01}$ & $\mathrm{X}, \mathrm{Fo} 06$ \\
\hline 45 & 3C 277.1 & 0.5 & & 8.0 & $1.28 \mathrm{E}-4$ & $-0.85_{-0.07}^{+0.07}$ & & & $\mathrm{C}, \mathrm{Si} 08$ \\
\hline 47 & $3 \mathrm{C} 281$ & 0.5 & & 7.0 & $1.00 \mathrm{E}-4$ & $-0.38_{-0.03}^{+0.04}$ & & & $\mathrm{C}, \mathrm{CSC}$ \\
\hline 48 & PG 1309+355 & 0.3 & 0.78 & 10.0 & $8.86 \mathrm{E}-5$ & $-1.92_{-0.13}^{+0.34}$ & $1.19 \mathrm{E}-4$ & $-0.73_{-0.05}^{+0.05}$ & X,Po04 \\
\hline 49 & PG $1322+659$ & 0.3 & 1.62 & 10.0 & $6.51 \mathrm{E}-4$ & $-2.01_{-0.11}^{+0.24}$ & $4.36 \mathrm{E}-4$ & $-1.18_{-0.11}^{+0.14}$ & X,Po04 \\
\hline 51 & PG $1351+640$ & 0.1 & & 2.4 & $1.41 \mathrm{E}-4$ & $-1.43_{-0.06}^{+0.06}$ & & & $\mathrm{R}, \mathrm{Yu} 98$ \\
\hline 53 & PG $1352+183$ & 0.3 & 2.00 & 10.0 & $7.15 \mathrm{E}-4$ & $-1.65_{-0.04}^{+0.04}$ & $4.34 \mathrm{E}-4$ & $-0.93_{-0.10}^{+0.10}$ & X,Po04 \\
\hline 54 & 4C 19.44 & 0.5 & 1.09 & 8.0 & $3.55 \mathrm{E}-4$ & $-0.88_{-0.28}^{+0.37}$ & $3.40 \mathrm{E}-4$ & $-0.39_{-0.09}^{+0.10}$ & $\mathrm{C}, \mathrm{Ga} 03$ \\
\hline 55 & 4C 58.29 & 0.1 & & 2.4 & $3.79 \mathrm{E}-5$ & $-1.62_{-0.72}^{+1.10}$ & & & $\mathrm{R}, \mathrm{Br} 97$ \\
\hline 56 & PG $1402+261$ & 0.3 & 1.73 & 10.0 & $9.01 \mathrm{E}-4$ & $-1.91_{-0.90}^{+0.13}$ & $6.04 \mathrm{E}-4$ & $-1.18_{-0.13}^{+0.08}$ & X,Po04 \\
\hline 57 & PG $1411+442$ & 0.2 & & 2.0 & $2.05 \mathrm{E}-5$ & $-1.97_{-0.07}^{+0.07}$ & & & $\mathrm{R}, \mathrm{La} 97$ \\
\hline 58 & PG $1415+451$ & 0.3 & 2.68 & 10.0 & $4.31 \mathrm{E}-4$ & $-1.92_{-0.09}^{+0.10}$ & $1.06 \mathrm{E}-4$ & $-0.50_{-0.20}^{+0.20}$ & $\mathrm{X}, \mathrm{Pi} 05$ \\
\hline 59 & PG 1425+267 & 0.2 & & 2.0 & $5.63 \mathrm{E}-5$ & $-0.94_{-0.16}^{+0.16}$ & & & $\mathrm{R}, \mathrm{La} 97$ \\
\hline 60 & PG $1427+480$ & 0.3 & 1.92 & 10.0 & $3.27 \mathrm{E}-4$ & $-1.53_{-0.03}^{+0.10}$ & $2.13 \mathrm{E}-4$ & $-0.87_{-0.07}^{+0.07}$ & X,Po04 \\
\hline 61 & PG 1440+356 & 0.3 & 1.56 & 10.0 & $1.80 \mathrm{E}-3$ & $-2.25_{-0.10}^{+0.10}$ & $1.22 \mathrm{E}-3$ & $-1.38_{-0.06}^{+0.06}$ & X,Po04 \\
\hline
\end{tabular}


Table 9

(Continued)

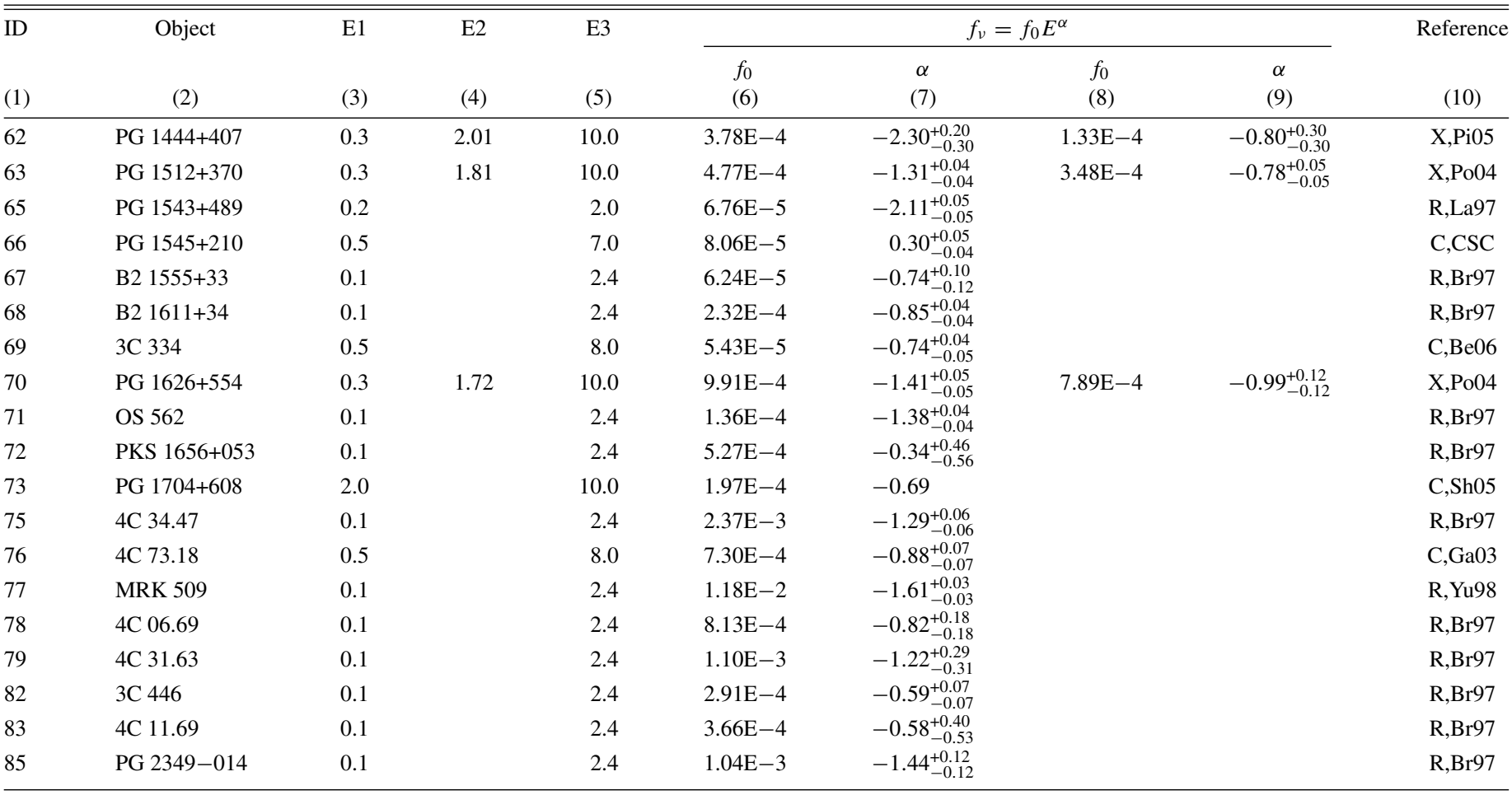

Notes. E1, E2, and E3 are the observed-frame energies (in keV) at which the power-law models are fit. When broken power-law models are used, E2 and Columns 8 and 9 are needed to present them. $f_{0}$ is flux density at $1 \mathrm{keV}$, in units of $\mathrm{mJy}\left(10^{-26} \mathrm{erg} \mathrm{s}^{-1} \mathrm{~cm}^{-2} \mathrm{~Hz}^{-1}\right) ; E$ in keV. Values without uncertainties reflect the fact that the original sources did not provide uncertainties.

References. R, C, and X indicate data sources, corresponding to ROSAT, Chandra, and XMM, respectively. Br97: Brinkmann et al. 1997; Yu98: Yuan et al. 1998; La97: Laor et al. 1997; Be06: Belsole et al. 2006; Ha96: Hardcastle et al. 2006; Sh05: Shi et al. 2005; Sa06: Sambruna et al. 2006; Ga03: Gambill et al. 2003; Ta07: Tavecchio et al. 2007; Si08: Siemiginowska et al. 2008; CSC: Chandra Source Catalog; Pi05: Piconcelli et al. 2005; Po04: Porquet et al. 2004; Fo06: Foschini et al. 2006.

Table 10

Parameters of Defining Bins in Each Waveband

\begin{tabular}{|c|c|c|c|c|c|c|c|c|}
\hline \multirow{2}{*}{$\begin{array}{l}\text { Wavelength } \\
\text { Band }\end{array}$} & \multicolumn{4}{|c|}{ Radio-loud } & \multicolumn{4}{|c|}{ Radio-quiet } \\
\hline & $v 1^{\mathrm{a}}$ & $v 2^{\mathrm{a}}$ & Bins $^{b}$ & $\Delta v^{\mathrm{c}}$ & $v 1^{\mathrm{a}}$ & $v 2^{\mathrm{a}}$ & Bins $^{b}$ & $\Delta v^{\mathrm{c}}$ \\
\hline Radio & 7.90 & 9.02 & 3 & 0.373 & 7.90 & 9.02 & 3 & 0.373 \\
\hline Radio & 9.18 & 9.55 & 1 & 0.370 & 9.13 & 9.51 & 1 & 0.380 \\
\hline Radio & 9.71 & 10.52 & 2 & 0.405 & 9.67 & 10.31 & 2 & 0.320 \\
\hline FIR & 12.33 & 12.89 & 2 & 0.280 & 12.28 & 12.81 & 2 & 0.265 \\
\hline MIR & 13.06 & 13.90 & 30 & 0.028 & 12.91 & 13.83 & 30 & 0.031 \\
\hline NIR & 14.20 & 14.54 & 4 & 0.085 & 14.15 & 14.49 & 3 & 0.113 \\
\hline UV/opt & 14.61 & 15.55 & 200 & 0.005 & 14.55 & 15.55 & 200 & 0.005 \\
\hline X-ray & 16.46 & 18.63 & 6 & 0.362 & 16.88 & 18.41 & 4 & 0.383 \\
\hline
\end{tabular}

Notes. See Section 5.2 on how the bins are defined in constructing composite SEDs.

${ }^{a}$ Frequency ranges in $\log (\mathrm{Hz})$.

b Number of bins in the range.

${ }^{\mathrm{c}}$ Bin size in $\log (\mathrm{Hz}), \Delta v=(v 2-v 1) /$ Bins.

we exclude this bin from the median SED. Figure 6 shows our median SEDs for RL and RQ samples.

\subsection{Discussion}

We try to keep all the original data in building the SEDs of individual objects. The only change is the resampling of the UV-optical spectra to $10 \AA$ resolution by rebinning. Although we lose some useful information (e.g., resolving narrow emission lines), this does not affect the SED work at all.

In constructing the composite SEDs, we applied rebinning again mostly for spectroscopic data. We did not apply any smoothing or interpolation in regions with real data, which could introduce systematic biases. The features in our median SEDs are real.

At the edges of some wavebands, the number of objects with data drops sharply (Figure 6, middle), and our method of using median to build the composite SEDs can help to some extent in the small number statistics. We also visually check to ensure that the SEDs are reasonably smooth in those regions.

\subsection{1. $R L$ versus $R Q$}

Figure 7 overplots our median SEDs of RL and RQ samples, normalized at $4215 \AA$ during the construction. The SEDs from FIR to UV are very similar for RL and RQ, especially in the UV-optical region. This is only true for the UV-optical continuum, because emission lines, such as Fe II and [O III], are known to strongly correlate with radio loudness in the Eigenvector I relationship (Boroson \& Green 1992). We will investigate the relationships between SEDs and emission line properties in a future paper.

The biggest difference between RL and RQ median SEDs is in the radio, where luminosity could differ by three orders of magnitude. There is also an obvious difference in the X-ray, where the RL objects are more X-ray luminous. This correlation between radio and X-ray luminosity has been reported in 

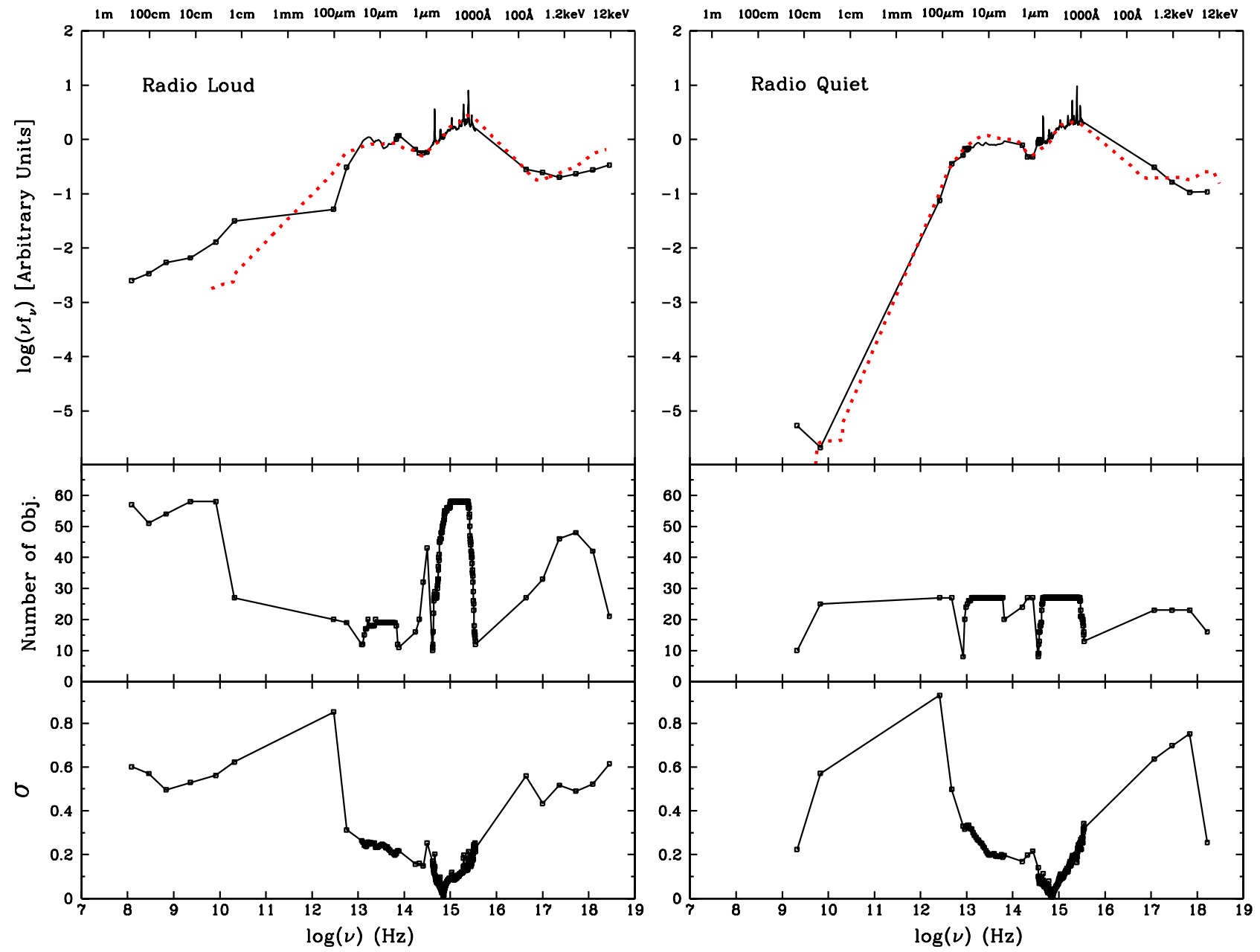

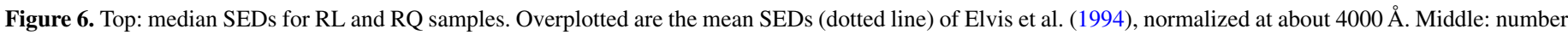

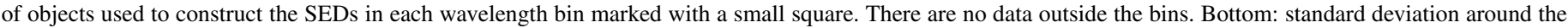
mean in each wavelength bin. The data of the median SEDs are available in the online version of the journal.

(A color version of this figure is available in the online journal. The data used to create this figure are available in the online journal.)

previous studies (e.g., Brinkmann et al. 2000; Polletta et al. 2007).

During the construction of the median SEDs, we have also defined six bins in the radio for the RQ sample (Table 10), however there are not enough objects $(\geqslant 8)$ in four of the bins. Therefore, there are only two points good enough to be included in the final radio SED of RQ sample. Given the big difference in the number of objects involved in these two bins (Figure 6), the apparent steep RQ spectral index, if defined using the two points, may not be reliable.

Our RL sample has more objects with higher redshifts than the RQ sample (Figure 1). We therefore build another median SED of RL objects only with redshift less than 0.5 (21 objects), similar to those in the RQ sample. Comparing this and the SED of the entire RL sample (Figure 7), we find that the only notable difference is in the radio where the low $-z$ subsample shows a less luminous radio SED, but radio spectral index seems similar. The difference is probably real because of sample propertieshigh- $z$ RL objects are more luminous in radio, but the differences between the RL and RQ samples are still much more prominent.

\subsubsection{Comparison with E94 Mean SEDs}

Elvis et al. (1994) use 47 objects to build the mean SEDs (MSED94) for RL and RQ objects. There are 11 objects in common with our sample, including $6 \mathrm{RL}$ and $5 \mathrm{RQ}$ objects. We compare our median SEDs of RL and RQ quasars (Figure 6) with MSED94. The overall shape of the SEDs over the entire available frequency range is similar, but there are more detailed features in our new SEDs.

We specifically keep all the emission features in the UV-optical region because they are real spectral features and our data quality allows us to keep them. The underlying continuum shapes in this region look similar to those of MSED94. We note that our UV-optical SEDs extend to shorter wavelength beyond $1000 \AA$, and start to turn over, indicating the peak of the "big blue bump" (e.g., Zheng et al. 1998; Shang et al. 2005). This is especially obvious in our RL median SED where we have more higher redshift objects.

In the MIR, the broad silicate emission features around 10 and $18 \mu \mathrm{m}$ are prominent in the SEDs. These could not otherwise be reproduced without the unprecedented spectral data from Spitzer IRS. To the shorter wavelength of these features, a welldefined power law rises up to about $4 \mu \mathrm{m}$, the IRS detecting limit for our sample in the rest frame. However, the well-known inflection around $1 \mu \mathrm{m}$ is also well defined by the red optical spectroscopy and NIR 2MASS photometry. It is therefore very clear that somewhere between 1 and $4 \mu \mathrm{m}$, there is an infrared bump, which is further supported by the fact that the NIR $K$-band data point starts to rise toward MIR in both RL and RQ SEDs. Other studies have suggested that there is a $3 \mu \mathrm{m}$ 


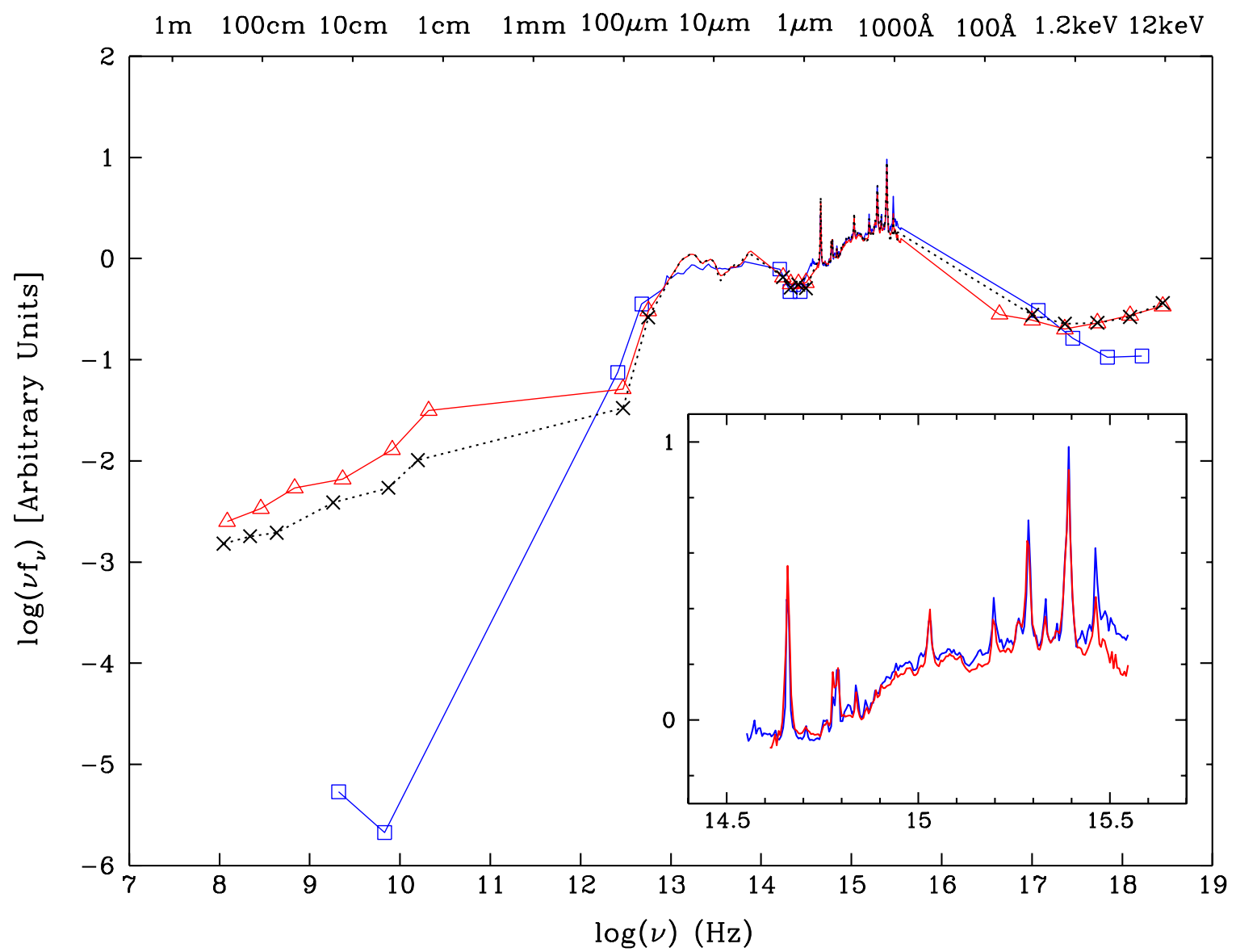

Figure 7. Median SEDs for the entire RL sample (open triangle, red) and RQ sample (open square, blue). Also shown is the median SED for only the low-redshift RL objects (cross, dotted line) for comparison (Section 5.3.1). The inset shows only the UV-optical region.

(A color version of this figure is available in the online journal.)

bump, resulting from the hottest dust in AGNs (Netzer et al. 2007; Hiner et al. 2009).

Although MSED94 have a lot of upper limits in the FIR from $I R A S$ while we use the latest Spitzer MIPS data, the SEDs agree surprisingly well for RQ sample in the FIR and extending to the radio.

For the RL sample, it is expected that our Spitzer data define a better FIR SED, which falls more steeply toward longer wavelengths. Our radio SED is more luminous than that of MSED94, simply because there are more radio-luminous objects in our sample.

\subsubsection{Comparison with Quasar SEDs of Richards et al. (2006)}

We compare our SEDs with those of Richards et al. (2006, hereafter R06) in Figure 8. R06 has provided the broadest frequency coverage, from Far-IR to X-ray, in recent SED studies, and their sample of 259 SDSS quasars extends to higher redshift and higher luminosity than ours. They constructed the SEDs using photometry points, including five SDSS magnitudes and four Spitzer Infrared Array Camera (IRAC) flux densities, supplemented by available GALEX $f$ and $n$ bands, $J, H$, and $K$, the ISO $15 \mu \mathrm{m}$ band, and the Spitzer MIPS 24 and $70 \mu \mathrm{m}$ bands. When objects do not have measurements in the supplemental bands, they used Elvis et al. (1994) SEDs and their "gap repair" technique to estimate the missing values. Their X-ray fluxes were obtained from ROSAT detections. When no detection is available, they estimated the X-ray flux using the $2500 \AA$ flux and the tight $L_{\mathrm{UV}}(2500 \AA)-L_{X}$ relationship (Strateva et al.
2005). They have only eight RL quasars, so the final SEDs are essentially for RQ quasars. Therefore, we only compare our RQ SED with theirs, but plot both of our RL and RQ SEDs in Figure 8 for completeness. We further choose to compare only with their two SEDs constructed with optically red and blue halves of their sample, and their mean SED would lie between the two.

Our SEDs show relatively much higher X-ray emission, indicating that our sample (and that of Elvis et al. 1994) is not representative of the SDSS quasars in this region and probably does not follow the $L_{\mathrm{UV}}(2500 \AA)-L_{X}$ relationship found in SDSS quasars (Strateva et al. 2005). In the far-IR to near-IR region, the overall shape and trend seem to match very well, but ours have more detailed features.

Although the SEDs are normalized at $4200 \AA$ (Figure 8), their shapes also match well over most of the UV-optical region. Only at the two ends, our SED show optical redder and UV brighter. This implies the different sample properties, because our objects are mostly UV-bright quasars and are probably redder in the $i$ band compared with R06 sample. Many objects in the R06 sample have much higher redshifts and luminosity than ours, but we do not have enough information to address any possible evolution or luminosity-dependent effects in quasar SEDs by comparing them.

\subsubsection{Comparison with Other Quasar Composites}

Figure 9 shows the comparison between our SEDs from farUV to mid-IR and other quasar composite spectra, including the 


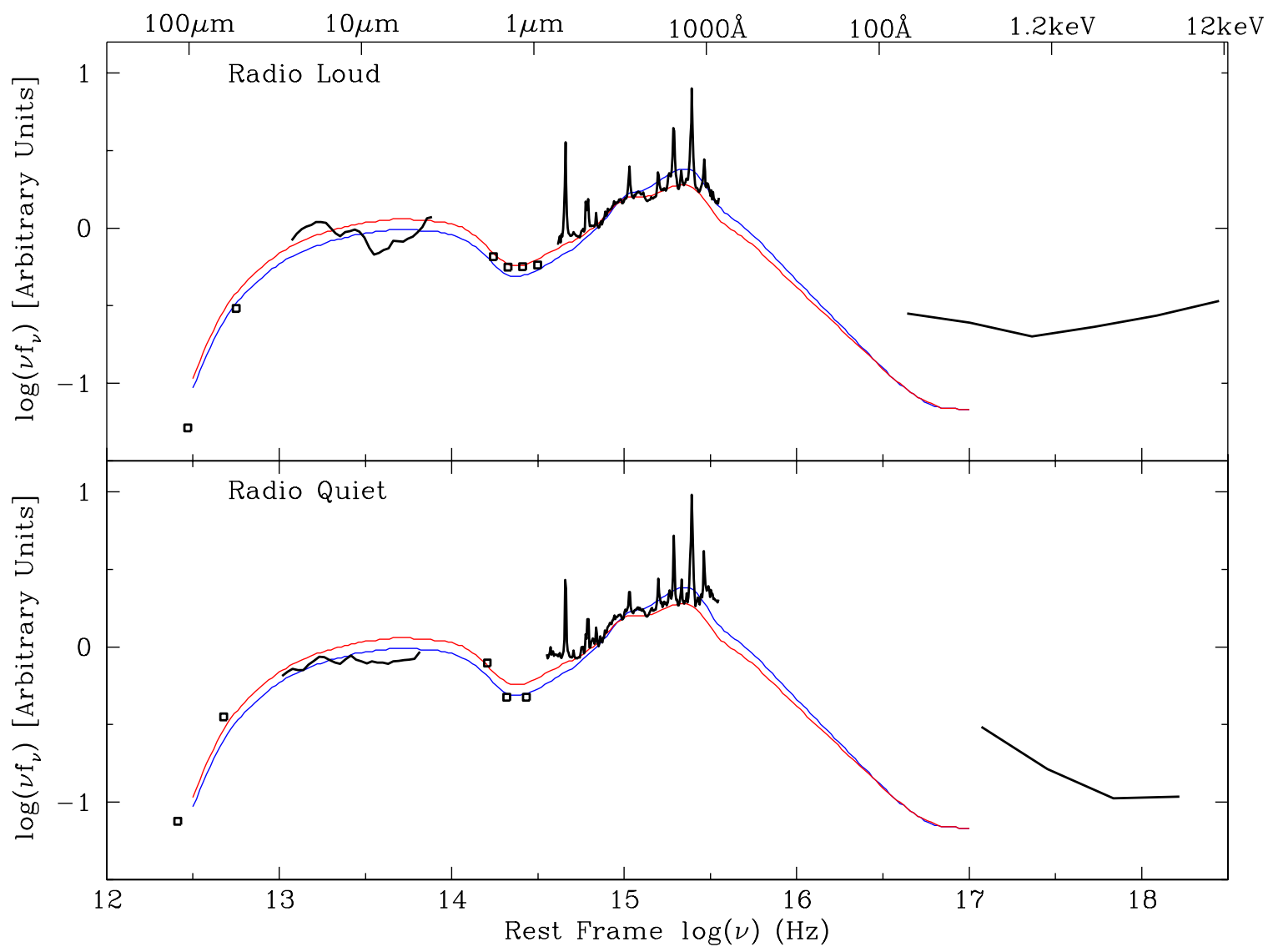

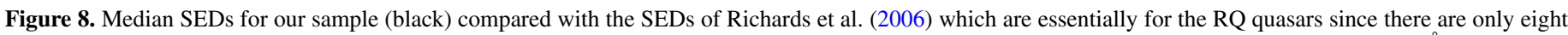
RL objects involved. The red and blue lines are for the optically red and blue halves of the population, respectively. The SEDs are normalized at $4200 \AA$.

(A color version of this figure is available in the online journal.)

HST UV composites (Telfer et al. 2002), the composite from Sloan Digital Sky Survey (SDSS; Vanden Berk et al. 2001), and a near-IR composite of 27 SDSS quasars (Glikman et al. 2006).

The HST composites extend beyond the FUV part of our SEDs to the extreme-UV (EUV), revealing the EUV peak more clearly. In the overlapping region, our SEDs are in good agreement with the HST composites although the RQ HST composite drops a little steeper at higher frequency. The SDSS composite includes both RL and RQ objects and is consistent with our SEDs between about 1200 and $4500 \AA$. The increased flux at the red part of the SDSS composite is partly due to host galaxy contamination at low redshift, while the blue part beyond Ly $\alpha$ should be ignored because of Ly $\alpha$ forest contamination. The NIR composites of RL and RQ samples show little difference in Glikman et al. (2006). We use the composite of their entire sample and its optical part matches our SEDs well. Its NIR region shows the $1 \mu \mathrm{m}$ inflection as expected, and the overall shape connects our MIR and optical SEDs surprisingly well. Although our NIR points are located a little lower than the composite, the continuum trends are consistent.

\subsection{Uncertainty and Caveats}

As in all previous quasar SED studies (e.g., E94; R06), the dispersion of the mean or composite SEDs is large. The dispersion of our median SEDs can be evaluated in Figure 6, where population standard deviations of all rebinned points in each bin are plotted at the bottom. Because we normalized the individual SEDs at $4215 \AA$, the dispersion is minimized in the optical and increases toward both low and high frequencies to about 0.6 (dex) in radio and X-ray. Even in the NIR to UV region, the standard deviation increases rapidly away from the normalization wavelength.

We also show the dispersion in Figure 10, where we plot all data from normalized SEDs of individual objects and the median SEDs built from them. The actual difference between the individual SEDs can be more than two orders of magnitude. Even in the NIR to UV, the difference is still more than one order of magnitude. These all indicate the large variation of quasar SEDs.

We note a caveat that the NIR host galaxy corrections may have a large uncertainty (Section 4.1, Table 6), because the $H$-band host galaxy measurements with ground-based infrared data may be somewhat inherently uncertain. The host galaxy fractions from HST observations (McLeod \& McLeod 2001, and our own observations) are systematically smaller than those from ground-based observations (McLeod \& Rieke 1994a, 1994b, and our IRTF data). Two objects have both ground-based and HST $H$-band observations and show revised lower host galaxy fractions from $H S T$ data (Table 6), with PG 1322+659 from $43 \%$ to $22 \%$, and PG $1427+480$ from $45 \%$ to $26 \%$, respectively. In addition, although the same technique was used in estimating the host galaxy fraction with all ground-based data, host galaxy fractions from McLeod \& Rieke (1994a) and our IRTF data seem to be systematically higher than McLeod \& Rieke (1994b) by about 20\%. Some SEDs show 


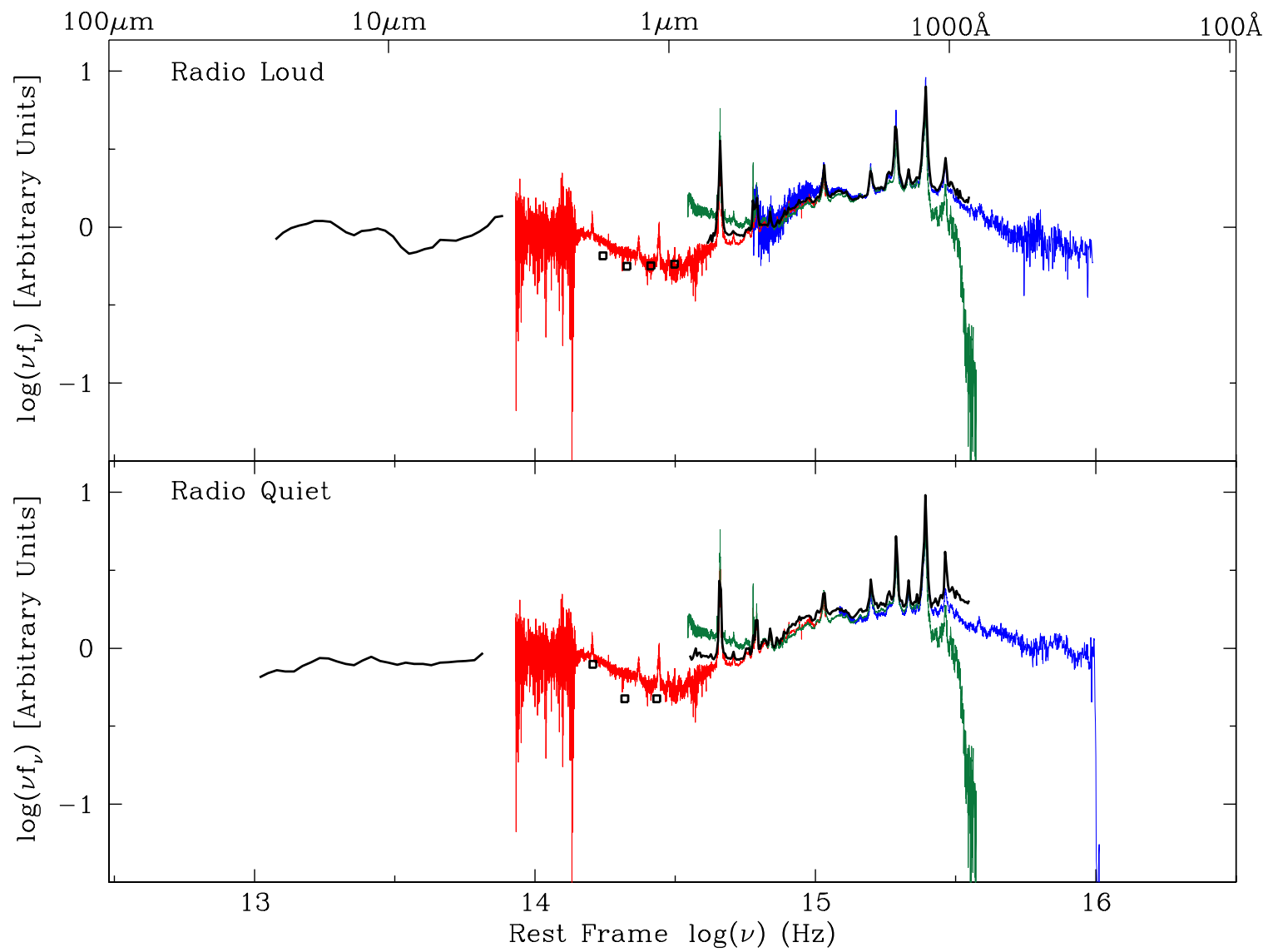

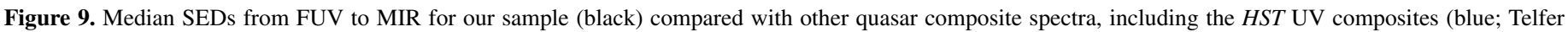

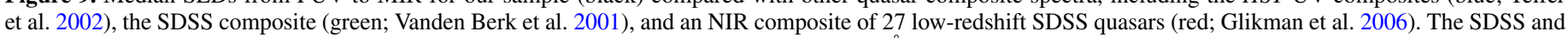
NIR composites do not distinguish RL or RQ objects. All composites are normalized at $4200 \AA$.

discrepancy between NIR broadband points and optical spectra (Figure 4), indicating possible over-subtraction of host galaxy contributions. Readers should be aware of this issue when using data of this region for their own applications.

Our sample is heterogeneous, and there is the possibility that the sample may be biased. Jester et al. (2005) pointed out that BQS quasars are representative of bright blue quasars, but not representative of bright red quasars. Since our sample involves many PG quasars, it is possible that we might be missing some red quasars, and therefore the SEDs are not truly representative of all UV-bright quasars. If this is true, it will affect the spectral index in the optical region. This does not seem to be a serious problem, however, because our SEDs do not show a drop-off in the red part of the optical region when compared with the SEDs of R06 (Figure 8). Moreover, even for the distinguished blue and red quasars in R06, except for the different spectral indices in the optical, their mean SEDs are very similar (see Figure 8, or their Figure 11), the largest difference in $\log \left(v f_{v}\right)$ is about 0.1 dex, significantly smaller than the dispersion of either R06 or our composite SEDs. Therefore, the large variation of individual quasar SEDs is still the dominant factor for the uncertainty of the composite SEDs.

\section{SUMMARY AND FUTURE WORK}

We have compiled SEDs for 85 quasars using high-quality multiwavelength data from radio to X-ray energies. The data were obtained from the next generation space telescopes and ground-based telescopes. Using these data, we have constructed composite (median) SEDs for RL and RQ quasars. This work is an update on the mean SEDs built by Elvis et al. (1994) with about twice as many objects. On our Web site ${ }^{16}$ and the online version of the journal, we make the electronic version of the median SEDs available for public use. We caution again that, because of the large variation in quasar SEDs, any composite SEDs should be used with care. Our composite SEDs are representative only for UV-optical bright quasars. The RQ median SED is constructed from low-redshift $(z<0.5)$ objects, while the RL median SED comes from objects of redshift up to 1.4 .

We also plan to investigate the multiwavelength data of individual objects. We have measured all the spectral parameters of the entire sample and the work will be presented in a separate paper. We will be able to obtain the bolometric luminosities from real data and investigate the bolometric corrections associated with spectral properties, such as continua or emission features. We have also planned to investigate how the quasar SED varies with different physical parameters such as black hole mass and Eddington ratio. The multiwavelength data will help us to better understand the quasars. A series of papers based on this data set are forthcoming.

This work has been supported by the National Natural Science Foundation of China through Grant No. 10773006 and Chinese 973 Program 2007CB815405. We are also grateful for support

\footnotetext{
16 http://physics.uwyo.edu/agn/
} 


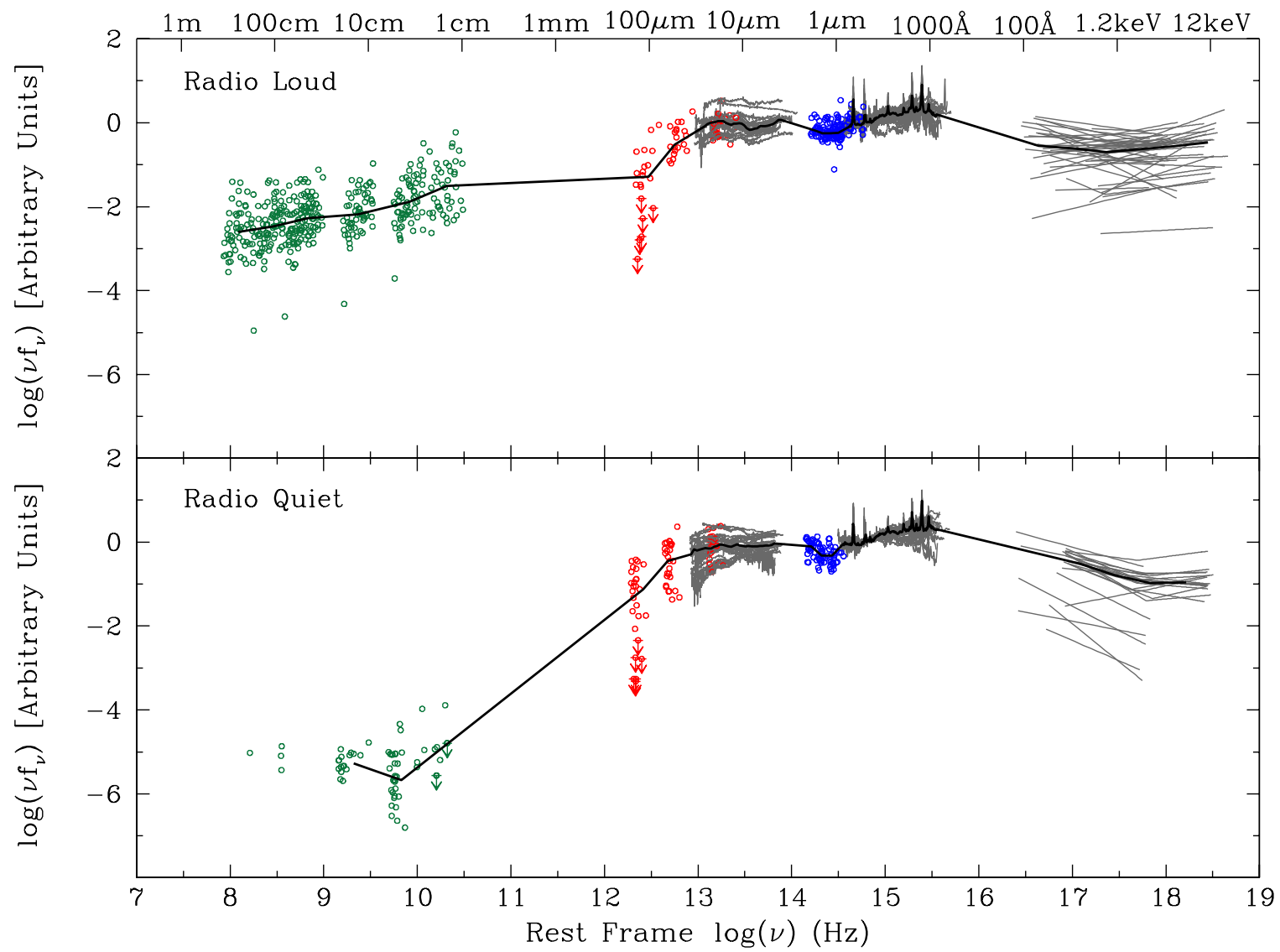

Figure 10. Dispersion of the median SEDs. All SEDs of individual objects, normalized at $4215 \AA$, are plotted together, showing large dispersions about the median SEDs (black thick line). Open circles are for radio (green), FIR (red), and NIR (blue) data. Spectroscopic data are shown in gray for MIR, UV/optical, and X-ray. (A color version of this figure is available in the online journal.)

by NASA through grant HST-GO-10717.01-A, Spitzer-GO20084, and Grant No. NNG05GD03G. B.J.W. acknowledges financial support by NASA through LTSA grant NAG5-3431 and HST grant GO-2578.01.87A, GO-4504, GO-5441, GO-06781, and AR-5820 from the Space Telescope Science Institute, which is operated by the Association of Universities for Research in Astronomy, Inc., under NASA contract NAS5-26555. We are also grateful for support from the US National Science Foundation, through grants AST-0206261, AST-8794137 (B.J.W.), and AST-0507781 (M.S.B.).

This publication makes use of data products from VLA, Spitzer, 2MASS, HST, FUSE, Chandra, XMM, ROSAT, and other facilities. We thank all those who built and operate them, who conducted the surveys, and who made available the data products and catalogs cited in this paper.

\section{REFERENCES}

Baldwin, J. A. 1977, ApJ, 214, 679

Barvainis, R., Lehár, J., Birkinshaw, M., Falcke, H., \& Blundell, K. M. 2005, ApJ, 618, 108

Barvainis, R., Lonsdale, C., \& Antonucci, R. 1996, AJ, 111, 1431

Becker, R. H., White, R. L., \& Helfand, D. J. 1995, ApJ, 450, 559

Belsole, E., Worrall, D. M., \& Hardcastle, M. J. 2006, MNRAS, 366, 339

Bolton, R. C., Cotter, G., Pooley, G. G., et al. 2004, MNRAS, 354, 485

Boroson, T. A., \& Green, R. F. 1992, ApJS, 80, 109 (BG92)

Brinkmann, W., Laurent-Muehleisen, S. A., Voges, W., et al. 2000, A\&A, 356, 445

Brinkmann, W., Yuan, W., \& Siebert, J. 1997, A\&A, 319, 413

Cardelli, J. A., Clayton, G. C., \& Mathis, J. S. 1989, ApJ, 345, 245

Cohen, A. S., Lane, W. M., Cotton, W. D., et al. 2007, AJ, 134, 1245

Condon, J. J., Cotton, W. D., Greisen, E. W., et al. 1998, AJ, 115, 1693
Dale, D. A., Gil de Paz, A., Gordon, K. D., et al. 2007, ApJ, 655, 863 Dale, D. A., Smith, J. D. T., Schlawin, E. A., et al. 2009, ApJ, 693, 1821 de Bruyn, G., Miley, G., Rengelink, R., et al. 1998, The Westerbork Northern Sky Survey, http://cdsarc.u-strasbg.fr/viz-bin/Cat?VIII/62

Diamond-Stanic, A. M., Rieke, G. H., \& Rigby, J. R. 2009, ApJ, 698, 623

Douglas, J. N., Bash, F. N., Bozyan, F. A., Torrence, G. W., \& Wolfe, C. 1996, AJ, 111,1945

Edelson, R. A., \& Malkan, M. A. 1986, ApJ, 308, 59

Elvis, M., Wilkes, B. J., McDowell, J. C., et al. 1994, ApJS, 95, 1

Evans, I. N., Primini, F. A., Glotfelty, K. J., et al. 2010, ApJS, 189, 37

Foschini, L., Ghisellini, G., Raiteri, C. M., et al. 2006, A\&A, 453, 829

Gallagher, S. C., Hines, D. C., Blaylock, M., et al. 2007, ApJ, 665, 157

Gambill, J. K., Sambruna, R. M., Chartas, G., et al. 2003, A\&A, 401, 505

Gear, W. K., Stevens, J. A., Hughes, D. H., et al. 1994, MNRAS, 267, 167

Genzel, R., Pauliny-Toth, I. I. K., Preuss, E., \& Witzel, A. 1976, AJ, 81, 1084

Glikman, E., Helfand, D. J., \& White, R. L. 2006, ApJ, 640, 579

Goulding, A. D., \& Alexander, D. M. 2009, MNRAS, 398, 1165

Gower, J. F. R., Scott, P. F., \& Wills, D. 1967, MmRAS, 71, 49

Griffith, M. R., Wright, A. E., Burke, B. F., \& Ekers, R. D. 1995, ApJS, 97, 347 Grupe, D., Komossa, S., Leighly, K. M., \& Page, K. L. 2010, ApJS, 187, 64

Hales, S. E. G., Riley, J. M., Waldram, E. M., Warner, P. J., \& Baldwin, J. E. 2007, MNRAS, 382, 1639

Hao, L., Spoon, H. W. W., Sloan, G. C., et al. 2005, ApJ, 625, L75

Hardcastle, M. J., Evans, D. A., \& Croston, J. H. 2006, MNRAS, 370, 1893

Hiner, K. D., Canalizo, G., Lacy, M., et al. 2009, ApJ, 706, 508

Houck, J. R., Roellig, T. L., van Cleve, J., et al. 2004, ApJS, 154, 18

Jester, S., Schneider, D. P., Richards, G. T., et al. 2005, AJ, 130, 873

Kellermann, K. I., \& Pauliny-Toth, I. I. K. 1973, AJ, 78, 828

Kellermann, K. I., Sramek, R., Schmidt, M., Shaffer, D. B., \& Green, R. 1989, AJ, 98, 1195

Kriss, G. A. 1988, ApJ, 324, 809

Kriss, G. J. 2000, in ASP Conf. Ser. 224, Probing the Physics of Active Galactic Nuclei, ed. B. M. Peterson, R. W. Pogge, \& R. S. Polidan (San Francisco, CA: ASP), 45

Kuraszkiewicz, J. K., Wilkes, B. J., Hooper, E. J., et al. 2003, ApJ, 590, 128 
Kuraszkiewicz, J., Wilkes, B. J., Schmidt, G., et al. 2009, ApJ, 692, 1143

Laor, A., Fiore, F., Elvis, M., Wilkes, B. J., \& McDowell, J. C. 1994, ApJ, 435, 611

Laor, A., Fiore, F., Elvis, M., Wilkes, B. J., \& McDowell, J. C. 1997, ApJ, 477, 93

Large, M. I., Cram, L. E., \& Burgess, A. M. 1991, Observatory, 111, 72

Large, M. I., Mills, B. Y., Little, A. G., Crawford, D. F., \& Sutton, J. M. 1981, MNRAS, 194, 693

Leipski, C., Falcke, H., Bennert, N., \& Huttemeister, S. 2006, A\&A, 455, 161

McLeod, K. K., \& McLeod, B. A. 2001, ApJ, 546, 782

McLeod, K. K., \& Rieke, G. H. 1994a, ApJ, 420, 58

McLeod, K. K., \& Rieke, G. H. 1994b, ApJ, 431, 137

McLeod, K. K., \& Rieke, G. H. 1995, ApJ, 454, L77

Moos, H. W., Cash, W. C., Cowie, L. L., et al. 2000, ApJ, 538, L1

Nemmen, R. S., \& Brotherton, M. S. 2010, MNRAS, 408, 1598

Netzer, H., Brotherton, M. S., Wills, B. J., et al. 1995, ApJ, 448, 27

Netzer, H., Lutz, D., Schweitzer, M., et al. 2007, ApJ, 666, 806

Peng, C. Y., Ho, L. C., Impey, C. D., \& Rix, H.-W. 2002, AJ, 124, 266

Pereira-Santaella, M., Diamond-Stanic, A. M., Alonso-Herrero, A., \& Rieke, G. H. 2010, ApJ, 725, 2270

Piconcelli, E., Jimenez-Bailón, E., Guainazzi, M., et al. 2005, A\&A, 432, 15

Pilkington, J. D. H., \& Scott, P. F. 1965, MmRAS, 69, 183

Polletta, M., Courvoisier, T. J.-L., Hooper, E. J., \& Wilkes, B. J. 2000, A\&A, 362,75

Polletta, M., Tajer, M., Maraschi, L., et al. 2007, ApJ, 663, 81

Porquet, D., Reeves, J. N., O’Brien, P., \& Brinkmann, W. 2004, A\&A, 422, 85

Rengelink, R. B., Tang, Y., de Bruyn, A. G., et al. 1997, A\&AS, 124, 259

Richards, G. T., Lacy, M., Storrie-Lombardi, L. J., et al. 2006, ApJS, 166, 470

Rieke, G. H., Young, E. T., Engelbracht, C. W., et al. 2004, ApJS, 154, 25

Risaliti, G., \& Elvis, M. 2004, in Supermassive Black Holes in the Distant Universe, ed. A. J. Barger (Astrophysics and Space Science Library, Vol. 308; Dordrecht: Kluwer), 187
Sambruna, R. M., Gliozzi, M., Tavecchio, F., Maraschi, L., \& Foschini, L. 2006, ApJ, 652, 146

Sanders, D. B., Phinney, E. S., Neugebauer, G., Soifer, B. T., \& Matthews, K. 1989, ApJ, 347, 29

Schlegel, D. J., Finkbeiner, D. P., \& Davis, M. 1998, ApJ, 500, 525

Schmidt, M., \& Green, R. F. 1983, ApJ, 269, 352

Schweitzer, M., Lutz, D., Sturm, E., et al. 2006, ApJ, 649, 79

Shang, Z., Brotherton, M. S., Green, R. F., et al. 2005, ApJ, 619, 41

Shang, Z., Wills, B. J., Robinson, E. L., et al. 2003, ApJ, 586, 52

Shang, Z., Wills, B. J., Wills, D., \& Brotherton, M. S. 2007, AJ, 134, 294

Shi, Y., Rieke, G. H., Hines, D. C., et al. 2005, ApJ, 629, 88

Siemiginowska, A., LaMassa, S., Aldcroft, T. L., Bechtold, J., \& Elvis, M. 2008, ApJ, 684, 811

Skrutskie, M. F., Cutri, R. M., Stiening, R., et al. 2006, AJ, 131, 1163

Strateva, I. V., Brandt, W. N., Schneider, D. P., Vanden Berk, D. G., \& Vignali, C. 2005, AJ, 130,387

Sun, W.-H., \& Malkan, M. A. 1989, ApJ, 346, 68

Tavecchio, F., Maraschi, L., Wolter, A., et al. 2007, ApJ, 662, 900

Telfer, R. C., Zheng, W., Kriss, G. A., \& Davidsen, A. F. 2002, ApJ, 565, 773

Tommasin, S., Spinoglio, L., Malkan, M. A., \& Fazio, G. 2010, ApJ, 709, 1257

Ulvestad, J. S., Antonucci, R. R. J., \& Barvainis, R. 2005, ApJ, 621, 123

Vanden Berk, D. E., Richards, G. T., Bauer, A., et al. 2001, AJ, 122, 549

Ward, M., Elvis, M., Fabbiano, G., et al. 1987, ApJ, 315, 74

Weedman, D. W., Hao, L., Higdon, S. J. U., et al. 2005, ApJ, 633, 706

Werner, M. W., Roellig, T. L., Low, F. J., et al. 2004, ApJS, 154, 1

Wilkes, B. J., Kuraszkiewicz, J., Green, P. J., Mathur, S., \& McDowell, J. C. 1999, ApJ, 513, 76

Wills, B. J., Thompson, K. L., Han, M., et al. 1995, ApJ, 447, 139

Yuan, W., Brinkmann, W., Siebert, J., \& Voges, W. 1998, A\&A, 330, 108

Zheng, W., Kriss, G. A., Telfer, R. C., Grimes, J. P., \& Davidsen, A. F. 1998, ApJ, 492, 855

Zheng, W., Kriss, G. A., Wang, J. X., et al. 2001, ApJ, 562, 152 